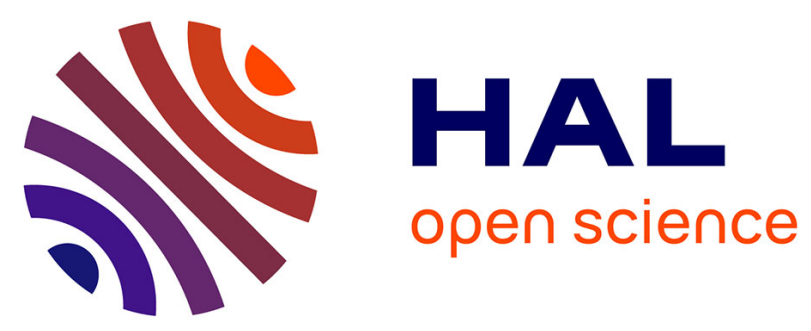

\title{
Practical review on the use of synchrotron based micro- and nano- X-ray fluorescence mapping and X-ray absorption spectroscopy to investigate the interactions between plants and engineered nanomaterials
} Hiram Castillo-Michel, Camille Larue, Ana Elena Pradas del Real, Marine Cotte, Geraldine Sarret

\section{To cite this version:}

Hiram Castillo-Michel, Camille Larue, Ana Elena Pradas del Real, Marine Cotte, Geraldine Sarret. Practical review on the use of synchrotron based micro- and nano- X-ray fluorescence mapping and $\mathrm{X}$-ray absorption spectroscopy to investigate the interactions between plants and engineered nanomaterials. Plant Physiology and Biochemistry, 2017, 110, pp.13 - 32. 10.1016/j.plaphy.2016.07.018 . hal-02325108

\section{HAL Id: hal-02325108 https://hal.science/hal-02325108}

Submitted on 10 Nov 2020

HAL is a multi-disciplinary open access archive for the deposit and dissemination of scientific research documents, whether they are published or not. The documents may come from teaching and research institutions in France or abroad, or from public or private research centers.
L'archive ouverte pluridisciplinaire HAL, est destinée au dépôt et à la diffusion de documents scientifiques de niveau recherche, publiés ou non, émanant des établissements d'enseignement et de recherche français ou étrangers, des laboratoires publics ou privés. 
Review

\title{
Practical review on the use of synchrotron based micro- and nano- X-ray fluorescence mapping and X-ray absorption spectroscopy to investigate the interactions between plants and engineered nanomaterials
}

\author{
Hiram A. Castillo-Michel ${ }^{\mathrm{a}, *}$, Camille Larue ${ }^{\mathrm{b}}$, Ana E. Pradas del Real ${ }^{\mathrm{a}, \mathrm{c}}$, Marine Cotte ${ }^{\mathrm{a}}$, Geraldine Sarret $^{\mathrm{c}}$ \\ a European Synchrotron Radiation Facility, Beamline ID21, Grenoble 38100, France \\ b ECOLAB, Université de Toulouse, CNRS, INPT, UPS, Toulouse, France \\ ' ISTerre (Institut des Sciences de la Terre), Université Grenoble Alpes, CNRS, Grenoble 38100, France
}

\section{A R T I C L E I N F O}

Article history:

Received 4 April 2016

Received in revised form 18 July 2016

Accepted 18 July 2016

Available online xxx

Keywords:

Synchrotron

X-ray mapping

Chemical speciation

\begin{abstract}
A B S T R A C T
The increased use of engineered nanomaterials $\left(\mathrm{ENM}_{\mathrm{S}}\right)$ in commercial products and the continuous development of novel applications, is leading to increased intentional and unintentional release of ENMs into the environment with potential negative impacts. Particularly, the partition of nanoparticles (NPs) to waste water treatment plant (WWTP) sludge represents a potential threat to agricultural ecosystems where these biosolids are being applied as fertilizers. Moreover, several applications of ENMs in agriculture and soil remediation are suggested. Therefore, detailed risk assessment should be done to evaluate possible secondary negative impacts. The impact of ENMS on plants as central component of ecosystems and worldwide food supply is of primary relevance. Understanding the fate and physical and chemical modifications of NPs in plants and their possible transfer into food chains requires specialized analytical techniques. Due to the importance of both chemical and physical factors to consider for a better understanding of ENMs behavior in complex matrices, these materials can be considered a new type of analyte. An ideal technique should require minimal sample preparation, be non-destructive, and offer the best balance between sensitivity, chemical specificity, and spatial resolution. Synchrotron radiation (SR) techniques are particularly adapted to investigate localization and speciation of ENMs in plants. SR X-ray fluorescence mapping (SR-XFM) offers multi-elemental detection with lateral resolution down to the tens of nm, in combination with spatially resolved X-ray absorption spectroscopy (XAS) speciation. This review will focus on important methodological aspects regarding sample preparation, data acquisition and data analysis of SR-XFM/ XAS to investigate interactions between plants and ENMs.
\end{abstract}

(C) 2016 Published by Elsevier Ltd.

\section{List of abbreviations}

ENMs

ESRF

EXAFS

$\mathrm{GeV}$

$\mathrm{keV}$

LCF

$\mathrm{LN}_{2}$

NNMA

NPs

OCT

PCA

PIXE

ROS

SR

TEM

\section{Engineered Nanomaterials}

European Synchrotron Radiation Facility

Extended X-ray Absorption Fine Structure

Gigaelectron Volt

Kiloelectron Volt

linear Combination Fitting

Liquid Nitrogen

Non-Negative Matrix Approximation

Nanoparticles

Optimal Cutting Temperature resin

Principal Component Analysis

Particle Induced X-ray Emission

Reactive Oxygen Species

Synchrotron Radiation

Transmission Electron Microscopy

\footnotetext{
* Corresponding author.

Email address: castillo@esrf.fr (H.A. Castillo-Michel)
}

$\begin{array}{ll}\text { WWTP } & \text { Waste Water Treatment Plants } \\ \text { XAS } & \text { X-ray Absorption Spectroscopy } \\ \text { XANES } & \text { X-ray Absorption Near-edge Structure } \\ \text { XRD } & \text { X-ray Diffraction } \\ \text { FTIR } & \text { Fourier Transform Infrared } \\ \text { SR-XFM } & \text { Synchrotron Radiation X-ray Fluorescence Mapping } \\ \text { XRF } & \text { X-ray Fluorescence. }\end{array}$

\section{Introduction}

Nanomaterials are defined as all materials that contain nanoscale structures (from 1 to $100 \mathrm{~nm}$ ). Due to their high surface area to volume ratios ( $>1 \%$ atoms at surface), they show different characteristics than the macro or micro sized materials such as high reactivity and conductivity. As the worldwide number of commercial products containing engineered nanomaterials (ENMs) is constantly increasing and new applications in many industrial fields (cosmetics, pharmacy, electronics, agriculture, etc.) are being developed every year, concentrations of these ENMs in ecosystems are likely to increase and reach levels with potential negative impacts. There could be intentional dissipation of ENMs from commercial products to the environment (e.g. 
nanofertilizers or nanopesticides), or unintentional release throughout their entire life cycle (e.g. by leaching from coatings or textiles (Benn and Westerhoff, 2008; Geranio et al., 2009; Kaegi et al., 2010)). Regardless of intention the release of ENMs and/or their derivatives could lead to negative impacts due to the potentially toxic properties of some ENMs. The most widely used ENMs are metallic and metal oxide nanoparticles (NPs) such as $\mathrm{TiO}_{2}, \mathrm{Ag}^{0}, \mathrm{CuO}, \mathrm{CeO}_{2}, \mathrm{Fe}_{\mathrm{x}} \mathrm{O}_{\mathrm{y}}$, and $\mathrm{ZnO}$ (Ma et al., 2015a). Probabilistic flow models predict environmental concentrations of widely used $\mathrm{NPs}\left(\mathrm{TiO}_{2}, \mathrm{Ag}^{0}\right.$, and $\left.\mathrm{ZnO}\right)$ accumulating mainly in wastewater treatment plant (WWTP) sludge followed by solid waste (Sun et al., 2014). For example, predicted concentrations of $\mathrm{TiO}_{2}$ and $\mathrm{ZnO}$ in WWTP sludge are $170 \mathrm{mg} / \mathrm{kg}$ and $24 \mathrm{mg} / \mathrm{kg}$, respectively for EU, and twice as much if the model was applied to Switzerland (Sun et al., 2014). Additionally, the predicted concentrations for $\mathrm{Ag}$ were of 0.02 and $0.04 \mathrm{mg} / \mathrm{kg}$ for EU and Switzerland, respectively. The partition of ENMs to WWTP sludge represents a potential threat to agricultural ecosystems where these biosolids are directly applied as fertilizers. About $50 \%$ of the total biosolids generated each year are used in agriculture and soil conditioning in Europe and the U.S. (Benn and Westerhoff, 2008; NEBRA, 2007). Hence, it is important to investigate the impact of ENMs from biosolid amendments in agricultural ecosystems. A recent study by Judy et al. (2015) described negative impacts to root nodulation frequency, plant growth, and increased metal uptake (Ti, $\mathrm{Zn}$ and $\mathrm{Ag}$ ) in Medicago truncatula G. plants. In addition, several applications of ENMs in agriculture (e.g. Ag nanopesticides and $\mathrm{ZnO}$ nanofertilizers) and soil remediation (e.g. zero valent Fe) are suggested. These applications could be of benefit but detailed studies should be done in order to evaluate possible secondary negative impacts (Hong et al., 2013; Martinez-Fernandez et al., 2016). Accumulation of ENMs in edible parts of crops, both from soil and foliar applications, could negatively impact human and livestock health (Hong et al., 2013; Rico et al., 2011). Understanding the fate and physical and chemical modifications of NPs in the environment, the impact of these changes on their bioavailability and their possible transfer in food chains is part of a new field of science called nanoecotoxicology.

Plants are a central component of ecosystems as primary producers, and are of main importance for the worldwide food supply. As all living organisms, plants have extremely complex mechanisms to regulate metal homeostasis in order to ensure optimal physiological and biochemical functions. Plants have evolved in presence of natural NPs consisting mainly (but not only) of oxides and oxyhydroxides of $\mathrm{Fe}, \mathrm{Mn}$ and $\mathrm{Al}$ and aluminosilicates present in soils (Hartland et al., 2013; Sharma et al., 2015). These natural NPs are prone to weathering processes induced by roots in the rhizosphere and used as nutrient source (Hinsinger, 1998). In other cases, plants are capable of producing natural NPs when in presence of exceeding amounts of ionic species (e.g. $\mathrm{Cu}, \mathrm{Ag}, \mathrm{Au}$ ), this is considered a defense mechanism to ionic toxicity (Sarret et al., 2013). However, the way plants will respond to increased concentrations of ENMs designed with specific properties to increase reactivity, stability, or release of derived products is an issue of debate. Current studies have evaluated the transfer of ENMs into plants, the toxicological impacts at both physiological and biochemical levels, and the chemical transformations they undergo in contact with plants. Recent reviews on the topic have presented a thorough summary of these findings (Ma et al., 2015a; Hossain et al., 2015; Schwab et al., 2016). From all these years of work some general lessons learned are paving the road to more relevant experimental designs. Uptake of NPs is plant species specific, highly influenced by growth conditions (matrix, temperature, light, exposure period, etc.) and particle size, shape, surface charge and functionalization; the same factors influence translocations to aerial tissues. At physiological level NPs impact plant morphology decreas- ing for example root length and biomass production, and altering the evapotranspiration rate. Symplast and apoplast are both possible routes of entrance involving both active and passive transport of NPs in plant tissues. Endocytosis, which is a well-recognized route for the entry of ENMs in animal cells, has been observed in a few studies in plants (Moscatelli et al., 2007; Bandmann et al., 2012; Wang et al., 2012). At biochemical level NPs increase the generation of reactive oxygen species (ROS) leading to lipid peroxidation and DNA damage. Excessive ROS induces scavenging enzymatic responses involving catalase, superoxide dismutase and/or ascorbic peroxidase, as well as the gluthatione biosynthesis pathway. Many of these responses to NPs are also triggered by elevated concentrations of some metal(loids). It is now well accepted that NPs elicit toxicity due to both ion release in the media and particle interactions with plant cell walls, blocking nutrient and water uptake, and leading to homeostatic imbalance (Martinez-Fernandez et al., 2016; Larue et al., 2016); or through the internationalization of NPs in plant cells followed by the release of high levels of toxic ions.

Over the years, knowledge regarding homeostatic balance of essential elements, as well as accumulation and detoxification of toxic elements in plants has increased through the use of analytical methods with improved detection limits, chemical sensitivity and spatial resolution (Lombi et al., 2011). Most of the analytical methods for studying elemental homeostasis are now being challenged by nanotechnology. As reviewed by (Laborda et al., 2016) ecotoxicological studies imply working with complex matrices (plants) and low concentrations. Due to the importance of both chemical (e.g. composition, oxidation state) and physical (e.g. size and shape) factors considering the understanding of ENMs behavior in complex matrices, these materials can be considered as a new type of analyte. In response to this challenge many analytical methods have been adapted, improved or developed. Commonly used techniques are based on light absorption/emission (e.g infrared, ultraviolet, visible and X-ray spectroscopy and microscopy), particle absorption/emission (e.g. electron microscopy and proton induced X-ray emission, PIXE), or rely on physical or chemical interactions (separation techniques e.g. field-flow fractionation and hydrodynamic chromatography). Since the NPs or their derived products (ions, or modified NPs) would impact differently plant specific functions depending on their location (at tissue and cellular level), agglomeration state, and/or chemistry, techniques that offer both spatial and chemical information are highly appreciated. An ideal technique should require minimal sample preparation (label free and no chemical extraction or fixation), should be non-destructive, and offer the best balance between sensitivity, chemical specificity, and spatial resolution. A recent review puts in focus the above mentioned aspects for some state-of-the-art imaging methods for metals in biology (Hare et al., 2015).

This review will focus on important methodological aspects of synchrotron X-ray fluorescence mapping (SR-XFM) and X-ray absorption spectroscopy (XAS) to elucidate the localization and speciation of NPs in plants. The objective is to present to the non-expert user the key steps for the optimal use of the techniques by revising critical steps of sample preparation, data acquisition and data analysis.

\subsection{Synchrotron techniques: localization and speciation}

The combined use of SR-XFM and XAS provide access to elemental composition, localization and chemical speciation offering a great balance of criteria for an ideal technique. From the acceleration of electrons to high energies (few $\mathrm{GeV}$ ) at almost the speed of light, extremely brilliant and highly collimated X-ray beams are produced at synchrotron radiation (SR) facilities. A SR X-ray source is at least 
11 orders of magnitude more intense than a laboratory X-ray source. New SR sources coming on-line and upgrade programs of present sources will produce more brilliant and coherent X-ray beams (Reich, 2013; De Jonge et al., 2014). Taking advantage of the brilliant X-ray source and their interactions with matter (absorption, emission, scattering) several techniques are offered at SR facilities. For an X-ray photon to be absorbed, its energy should be higher than the binding energy of an electron in an atomic orbital (binding energy of $\mathrm{K}>\mathrm{L}>\mathrm{M}$ orbitals). In SR-XFM applications, X-rays produced at a SR source are carefully selected and focused at specialized experimental stations called beamlines. X-ray photons shine on the samples ejecting core electrons from atomic orbitals, the created holes are then filled by electrons from higher orbitals. During this relaxation process, X-ray photons called fluorescence photons are emitted. These photons have characteristic energies for each element in the periodic table and are detected by solid state detectors to infer the elemental composition in the illuminated specimen area. XAS is a technique that interprets the features produced by the modulation of the absorption coefficient across the binding energy of a core electron. It is an element specific spectroscopic technique that provides information about the oxidation state and local geometry of the absorbing element. For XFM the use of SR makes possible to reach high spatial resolution (few $\mu \mathrm{m}$ to tens of $\mathrm{nm}$ ) and low detection limits (femtograms) in reasonable acquisition times $(\leq 1 \mathrm{~s}$ per pixel). In the case of XAS, achieving good detection limits (picograms) and the required spectral resolution (sub-eV) makes this technique almost exclusive of synchrotron facilities (Schlesiger et al., 2015). Considering the analysis is performed in very small sample volumes (e.g. $1 \mu \mathrm{m}^{2}$ beam illuminates $25-40 \mu \mathrm{m}$ thick plant sample) SR-XFM can detect down to femtograms of the element of interest, depending on flux, detector efficiency, and matrix of the sample. Assuming density of plant tissue close to water, femtogram content of the element of interest would translate to $\sim 10-100 \mathrm{mg} / \mathrm{kg}$ local concentration in the tissue. For example, this concentration level could be reached by one $80-100 \mathrm{~nm} \mathrm{Ag} \mathrm{NP} \mathrm{(mass} \mathrm{of} \sim 1 \times 10^{14} \mathrm{~g}$ ) present in $25 \mu^{3}$ sample volume. As a rule of thumb XAS of exploitable quality requires at least a factor of 10 higher concentrations.

These techniques are well known in environmental sciences for their applicability to investigate elemental content and speciation in complex matrices (Majumdar et al., 2012; Pushie et al., 2014). In fact, SR techniques have provided fundamental knowledge in a myriad of studies involving plants and metal(loid) homeostasis (Sarret et al., 2013). Naturally, the techniques are now becoming equally popular and relevant, due to improvements in detection limits and spatial resolution, in the study of plant/ENMs interactions. Recent reviews have focused or dedicated part of their discussion to illustrate applications of SR-XFM and XAS in the study of metals in plants including the cases where metals are in NP form (Rico et al., 2011; Majumdar et al., 2012; Sarret et al., 2013; Grafe et al., 2014; Laborda et al., 2016; Ma et al., 2015a,b). The combination of SR techniques able to provide information at different scales is quite useful. For example, starting by investigating the organ level (e.g. roots, shoots) speciation by non-spatially resolved XAS is an efficient first approach to elucidate chemical interactions of NPs with plants (e.g. oxidation state change or binding to $\mathrm{O}$ or $\mathrm{S}$ ligands). Further, characterization at the micron scale by SR- $\mu$ XFM/XAS for localization and speciation at the tissue level (e.g epidermis, cortex, stele) can provide information on the fate of NPs and released ions in each compartment. Ultimately, the use of SR-nanoXFM/XAS allows the observation of smaller aggregate and individual particle distribution and chemical state. Though resolution at the nanoscale is better achieved with transmission electron microscopy (TEM), SR-nanoXFM is more informative due to its multi-elemental nature combined with the chemical sensitivity of XAS. Recently, this combination of techniques has been used to provide a detailed study about the fate of Ag NPs in agricultural soils (Pradas del Real et al., 2016). In plants the combination of SR- $\mu$ XFM/XAS and SR-nanoXFM allowed the observation of Ag NPs in the vascular region (Fig. 1A, D, and E) of sunflower (Helianthus annuss L.) roots, and the identification of Ag coordiantion to $\mathrm{S}$ ligands at the casparian strip (Fig. 1C) (Martinez-Criado et al., 2015). This experiment required the use of 2 beamlines operating at different excitation energies allowing SR- $\mu$ XFM/XAS at Ag $\mathrm{L}_{\text {III }}$-edge (with simultaneous detection of $\mathrm{Cl}, \mathrm{S}$, and $\mathrm{P}$; see Fig. 1B), and SR-nanoXFM at Ag K-edge (Fig. 1D and E). NanoXAS was at the time of the experiment under development at ID16B; this technique is now available to users. As illustrated by this example, this type of experimental approach is useful but it requires access to multiple beamlines and/or SR facilities so the experiment should be carefully planned to comply with beamline specifications and timing.

Since synchrotron access is given via peer-reviewed experimental proposals, it is always recommended to have a good characterization of the samples at the macroscopic/tissue level (e.g. total concentrations from inductively coupled plasma mass spectrometry (ICP-MS) analyses). Experimental proposals are typically 2-3 page documents that should state the scientific relevance of the experiment, a maximum of technical details (number of samples, sample preparation, data acquisition, and data analysis), and a good justification of the selected beamline and of the requested time for experiments. Most facilities receive proposals twice a year. SR facilities operate 24/24 for 6 continuous days, the user group must be prepared to take full advantage of an experimental session (typically 4-6 days) this implies working night shifts. A useful description of the important criteria for selecting the appropriate beamline, applying for beamtime, and planning an experiment is provided by Donner et al. (2013). The composition of the ENM under investigation, type of information desired (elemental distribution and/or speciation) and target scale (tissue, cell or sub-cell level), as well as the required level of preservation of the sample will determine the choice of a beamline where experiments could be carried out most effectively (see Fig. 2). This is discussed more in detail in the coming sections that will cover important aspects regarding sample preparation, data acquisition, and data analysis.

\section{Sample preparation}

Sample preparation is a critical step for imaging techniques such as SR-XFM and even more critical for techniques focusing on elemental speciation such as XAS (Sarret et al., 2013). These spatially resolved techniques are preferentially applied on plant tissue cross-sections in order to distinguish the signal coming from specific tissues or cells. The lateral resolution of these techniques is defined by the beam size achieved by the X-ray optics. However, due to the high penetration depth of X-rays, the sample thickness would define the probed volume providing average information on several cell layers. Still, visualizing the distribution of elements at the organ scale by working on whole leaves or roots also presents some interests, particularly for 3D SR-XFM applications (De Jonge et al., 2014). For SR-XFM, sample preparation protocols should essentially preserve elemental distribution. For XAS, sample preparation should preserve the chemical form of the element of interest.

Due to the high water content of plants, which accounts for $40-90 \%$ of their tissues, handling water appropriately is one of the main issues in sample preparation. For SR-XFM applications samples can be analyzed under two conditions: dehydrated vs. hydrated state. 


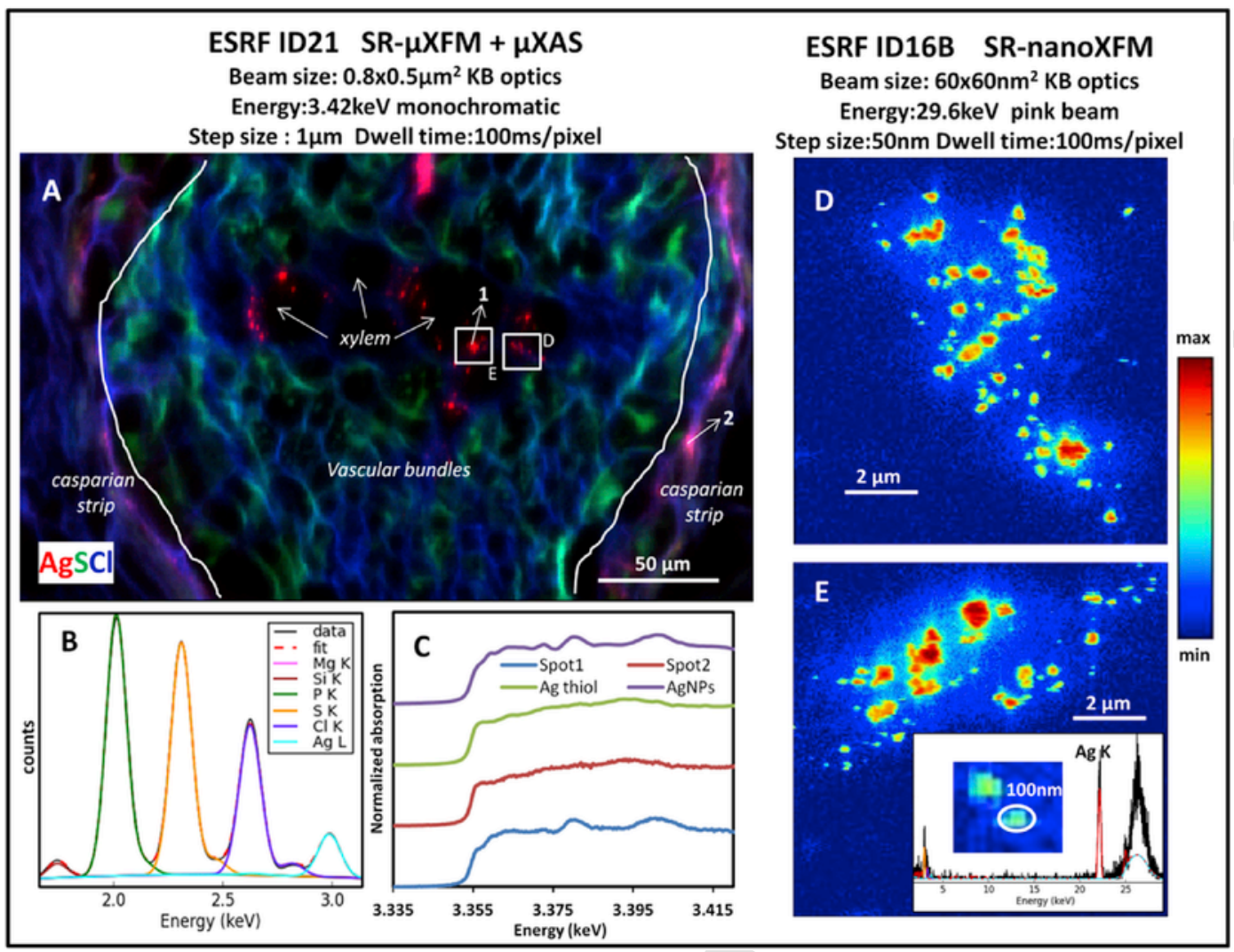

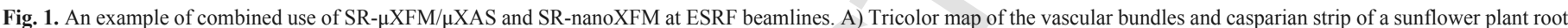

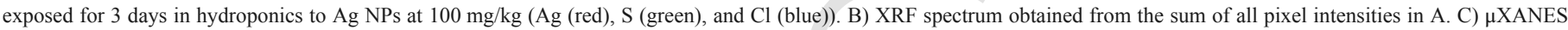

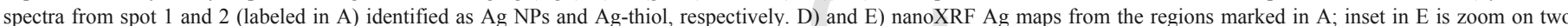

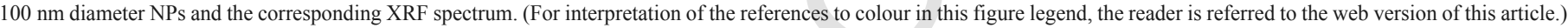

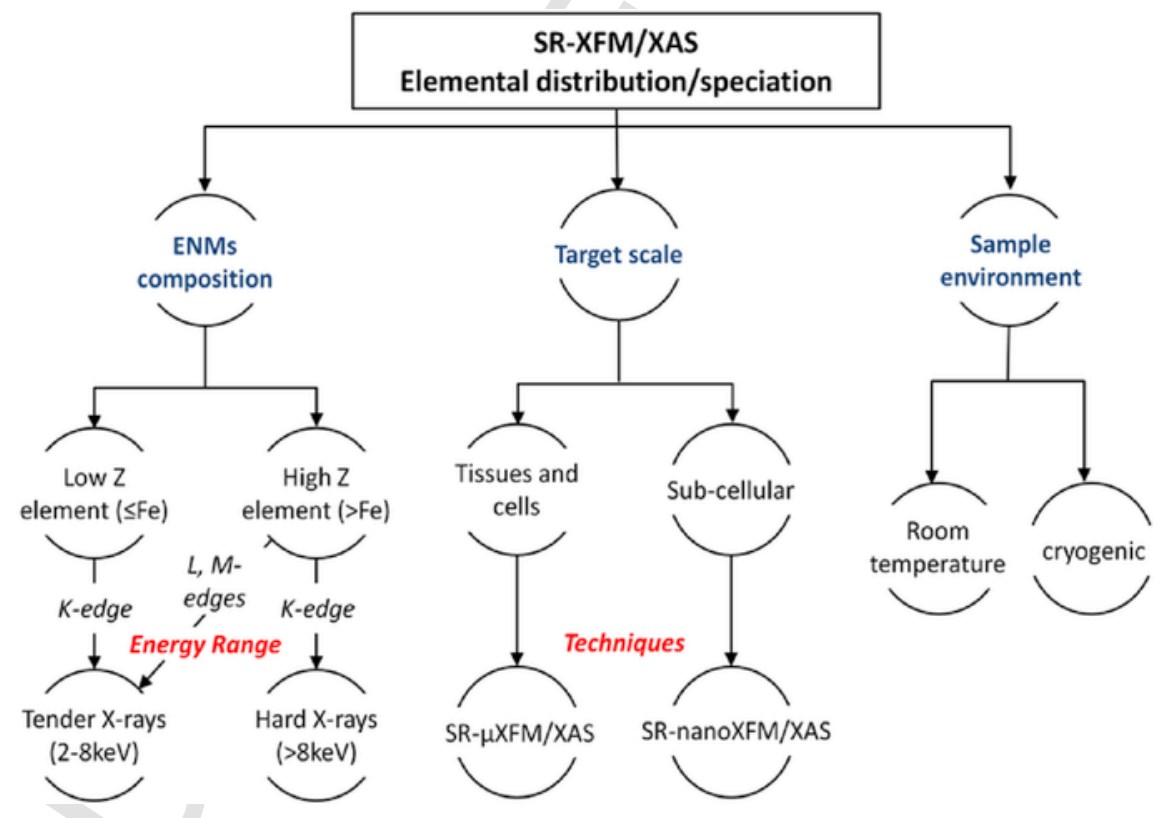

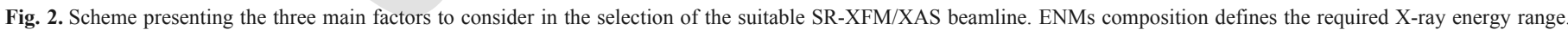

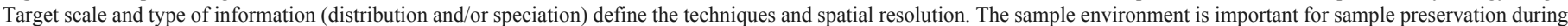
analyses.

Hydrated plant tissues can be analyzed either in their native state using a very fast and sensitive detector (such as Maia detector (Wang et 
al., 2013)) - in that case no sample preparation is required - or in frozen hydrated state using a cryogenic system (Salome et al., 2013). Hence, the conditions for analysis are not only defined by the user but are somehow determined by the equipment available at the beamlines (e.g. type of detectors and cryogenic stage). In this section, we present the protocols employed to prepare samples for SR-XFM/XAS applications (Fig. 3). Most of the protocols described here have been originally developed for electron microscopy and adapted for SR techniques. In the literature, 23 studies focused on elemental distribution and speciation of NPs in plants have been listed in which 14 have been performed on freeze-dried samples, four on frozen-hydrated samples, and two on chemically fixed samples (see Table 1).

\subsection{Chemical fixation}

Chemical fixation is a common sample preparation technique for TEM analysis. This protocol includes several steps: (i) fixation of sample tissue by creating inter and intramolecular bridges using chemical fixatives, (ii) sample dehydration through several baths with increasing ethanol concentrations and (iii) inclusion in a hydrophobic resin.

Several tests have been performed to compare elemental distribution after chemical fixation using different types of chemicals vs. distribution in frozen hydrated samples. Formalin has been tested on both animal and human tissues (but not for plant samples) and seems to be the least favorable option for fixation with leaching of components such as amino acids, carbohydrates, lipids, proteins, and ions. It also induces redistribution within the sample of $\mathrm{K}, \mathrm{Ca}, \mathrm{Fe}, \mathrm{Cu}$ and Zn (Chwiej et al., 2005; Hackett et al., 2011; Punshon et al., 2015; Zohdi et al., 2015). Recently, Perrin et al. (2015) reported poor preservation of intracellular ions such as $\mathrm{Mg}$ and $\mathrm{K}$ after paraformaldehyde or methanol chemical fixation in PC12 cells. The authors compared chemical fixation vs cryofixation and report a decrease of $\mathrm{K}$ concentration by a factor of one hundred for the chemically fixed samples. Moreover, an increase in $\mathrm{S}$ and $\mathrm{Zn}$ concentrations in cells fixed using paraformaldehyde was observed and is probably a contribution from the fixation solution. Other often used fixative chemicals are formaldehyde and glutaraldehyde in sodium cacodylate buffer. Hansson et al. (2008) demonstrated that in thyroid, chemically fixed samples had dramatically decreased iodine content ( -14 to $30 \%$ ) in comparison to frozen hydrated samples. However, other articles demonstrated that this association of chemicals gave better results than formalin (Punshon et al., 2015) and even comparable results to unfixed samples in the case of the study of Fe distribution in Arabidopsis thaliana seeds (Punshon et al., 2012).

Two studies found in the literature have used chemical fixation protocols to investigate interactions between plants and NPs by SR-XFM (Sabo-Attwood et al., 2012). reported the use of chemical fixation to localize and analyze the speciation of Au-NPs in Nicotiana xanthi L.. Au NPs of $3.5 \mathrm{~nm}$ were detected in plants and no chemical modification was reported. The second study focused on the distribution of three different forms of $\mathrm{Ag}$ ( $\mathrm{Ag} \mathrm{NPs}, \mathrm{Ag}_{2} \mathrm{~S} \mathrm{NPs}$, and ionic $\mathrm{Ag}$ ) in roots of Medicago sativa (Stegemeier et al., 2015). This study revealed different accumulation patterns for the tested $\mathrm{Ag}$ forms, $\mathrm{Ag}$ NPs accumulated mainly in the columella cells, ionic Ag was more uniformly distributed throughout the root, and $\mathrm{Ag}_{2} \mathrm{~S}$ NPs remained largely adhered to the root exterior $\left(\mathrm{Si}_{\mathrm{x}} \mathrm{O}_{\mathrm{x}} \mathrm{NPs}\right.$ were also observed in cell cytoplasm). In both studies the resolution used for SR- $\mu$ XFM does not allow to judge the sample preparation protocol at cellular level.

The overall conclusion is that chemical fixation may not be an ideal strategy; although it usually preserves the ultrastructure of the sample, it can lead to elemental redistribution and loss of content. Moreover, the protocol is composed of several handling steps which increase the risks of damaging or contaminating the samples. More comparative studies between chemically fixed and frozen hydrated samples in plants would be useful for validation of adequate fixation protocols. The underlying mechanisms of chemical fixation (bridging molecules) make this protocol not recommended for speciation analyses.

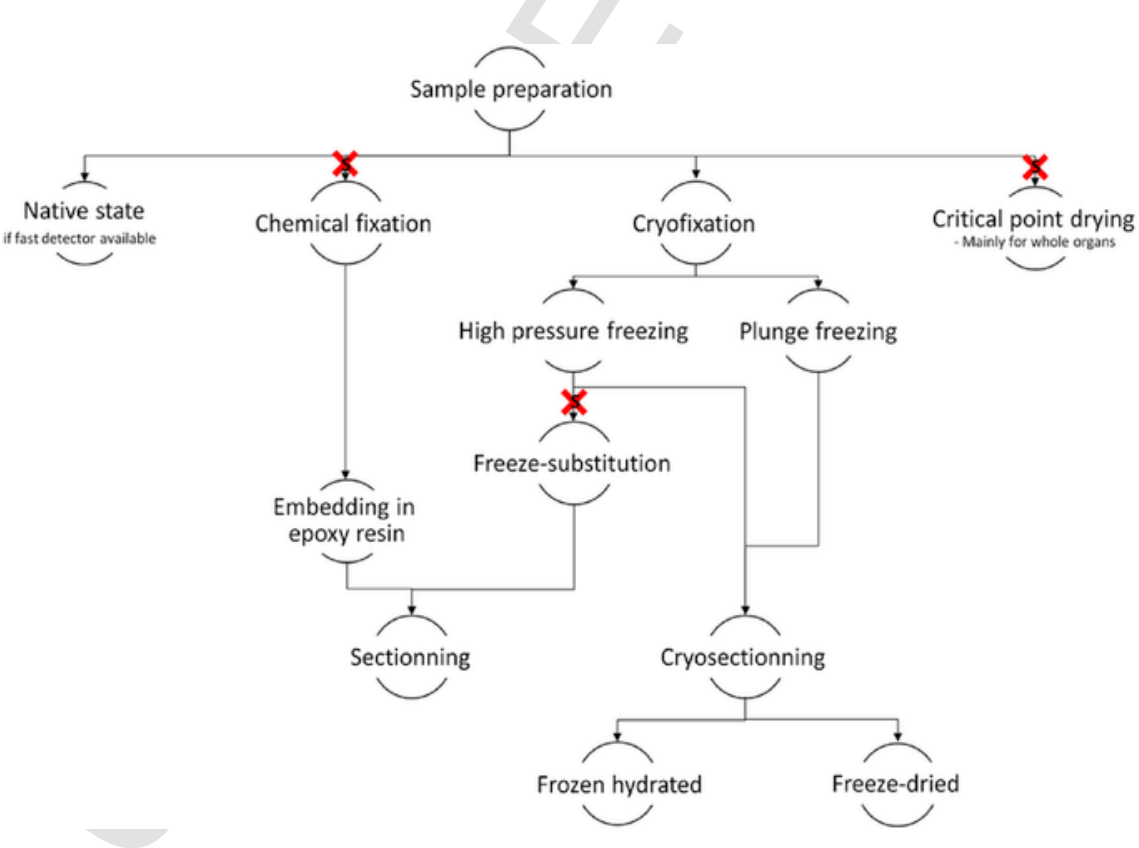

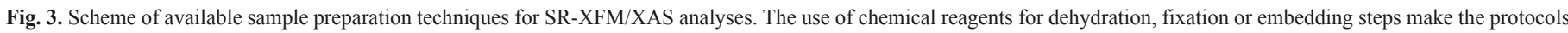
marked with $\times$ not recommended for speciation experiments. 
Table 1

Sample preparation protocols used in the literature for SR-XFM/XAS studies

\begin{tabular}{|c|c|c|c|}
\hline Techniques & Sample preparation & $\begin{array}{l}\text { Plant species } \\
\text { (tissue) }\end{array}$ & $\begin{array}{l}\text { NPs (cultivation } \\
\text { media) }\end{array}$ \\
\hline $\begin{array}{l}\text { SR- } \mu \text { XFM/ } \mu \text { XAS Zn K- } \\
\text { edge (Hernandez-Viezcas } \\
\text { et al., 2011) }\end{array}$ & $\begin{array}{l}\text { Plunge-freezing } \\
45 \text { min in } \mathrm{LN}_{2} \\
\text { Embedded in OCT } \\
\text { compound } \\
\text { Cryosectioned } 30 \\
\text { and } 100 \mu \mathrm{m} \text { thick } \\
\text { Freeze-dried } 3 \\
\text { days }\left(-53^{\circ} \mathrm{C} \text {, }\right. \\
0.140 \mathrm{mbar})\end{array}$ & $\begin{array}{l}\text { Velvet } \\
\text { mesquite } \\
\text { Prosopis } \\
\text { juliflora- } \\
\text { velutina } \text { sw. } \\
\text { (Root and } \\
\text { leaf) }\end{array}$ & $\begin{array}{l}\mathrm{ZnO} \\
\text { (Hydroponics) }\end{array}$ \\
\hline $\begin{array}{l}\text { SR- } \mu \text { XFM/ } \mu \text { XAS Ag } \\
\mathrm{L}_{\mathrm{III}} \text {-edge (Yin et al., } \\
2011)\end{array}$ & $\begin{array}{l}\text { Cryosectioned } \\
55 \mu \mathrm{m} \text { thick }\end{array}$ & $\begin{array}{l}\text { Ryegrass } \\
\text { Lolium } \\
\text { multiflorum } \\
\text { L. } \\
\text { (Root) }\end{array}$ & $\begin{array}{l}\mathrm{Ag} \\
\text { (Hydroponics) }\end{array}$ \\
\hline $\begin{array}{l}\text { SR- } \mu \text { XFM/ } \mu \text { XAS Ti K- } \\
\text { edge (Larue et al., 2012a) }\end{array}$ & $\begin{array}{l}\text { Plunge-freezing } \\
\text { (isopentane) } \\
\text { Embedded in OCT } \\
\text { compound } \\
\text { Cryosectioned } \\
50 \mu \mathrm{m} \text { thick } \\
\text { Freeze-dried }\end{array}$ & $\begin{array}{l}\text { Wheat } \\
\text { Triticum } \\
\text { aestivum L. } \\
\text { Rapeseed } \\
\text { Brassica } \\
\text { napus L. } \\
\text { (Root and } \\
\text { leaf) }\end{array}$ & $\begin{array}{l}\mathrm{TiO}_{2} \\
\text { (Hydroponics) }\end{array}$ \\
\hline $\begin{array}{l}\text { SR- } \mu \text { XFM/ } \mu \text { XAS Ti K- } \\
\text { edge (Larue et al., 2012b) }\end{array}$ & $\begin{array}{l}\text { Plunge-freezing } \\
\text { (isopentane) } \\
\text { Embedded in OCT } \\
\text { compound } \\
\text { Cryosectioned } \\
50 \mu \mathrm{m} \text { thick } \\
\text { Freeze-dried } 48 \mathrm{~h} \\
\left(-10^{\circ} \mathrm{C} \text {, }\right. \\
0.37 \mathrm{mbar})\end{array}$ & $\begin{array}{l}\text { Wheat } \\
\text { Triticum } \\
\text { aestivum L. } \\
\text { (Root and } \\
\text { leaf) }\end{array}$ & $\begin{array}{l}\mathrm{TiO}_{2} \\
\text { (Hydroponics) }\end{array}$ \\
\hline $\begin{array}{l}\text { SR- } \mu \text { XFM/ } \mu \text { XAS Au } \\
\mathrm{L}_{\mathrm{III}} \text {-edge (Sabo-Attwood } \\
\text { et al., 2012) }\end{array}$ & $\begin{array}{l}\text { Infiltrated by a } \\
\text { hydrophilic glycol } \\
\text { methacrylate resin } \\
\text { Sectioned } 100 \mu \mathrm{m} \\
\text { thick }\end{array}$ & $\begin{array}{l}\text { Tobacco } \\
\text { Nicotiana } \\
\text { xanthi L. } \\
\text { (Root and } \\
\text { leaf) }\end{array}$ & $\begin{array}{l}\mathrm{Au} \\
\text { (Hydroponics) }\end{array}$ \\
\hline $\begin{array}{l}\text { SR- } \mu \text { XFM/ } \mu \text { XAS Ti K- } \\
\text { edge (Servin et al., 2012) }\end{array}$ & $\begin{array}{l}\text { Plunge-freezing in } \\
\mathrm{LN}_{2} \\
\text { Embedded in OCT } \\
\text { compound } \\
\text { Cryosectioned } \\
30 \mu \mathrm{m} \text { thick } \\
\text { Freeze-dried }\end{array}$ & $\begin{array}{l}\text { Cucumber } \\
\text { Cucumis } \\
\text { sativus L. } \\
\text { (Root and } \\
\text { leaf) }\end{array}$ & $\begin{array}{l}\mathrm{TiO}_{2} \\
\text { (Hydroponics) }\end{array}$ \\
\hline $\begin{array}{l}\text { SR- } \mu \text { XFM/ } \mu \text { XAS Ce } \\
\mathrm{L}_{\text {III-edge (Zhao et al., }} \\
2012 \text { ) }\end{array}$ & $\begin{array}{l}\text { Plunge-freezing in } \\
\mathrm{LN}_{2} \\
\text { Embedded in OCT } \\
\text { compound } \\
\text { Cryosectioned } \\
30 \mu \mathrm{m} \text { thick } \\
\text { Freeze-dried } 48 \mathrm{~h} \\
\left(-53^{\circ} \mathrm{C} \text {, }\right. \\
0.140 \mathrm{mbar})\end{array}$ & $\begin{array}{l}\text { Maize } \\
\text { Zea mays L. } \\
\text { (Root) }\end{array}$ & il) \\
\hline $\begin{array}{l}\text { SR- } \mu \text { XFM/ } \mu \text { XAS Ce } \\
\mathrm{L}_{\mathrm{III}} \text {-edge, Zn K-edge } \\
\text { (Hernandez-Viezcas et } \\
\text { al., 2013) }\end{array}$ & $\begin{array}{l}\text { Plunge-freezing } \\
30 \text { min in } \mathrm{LN}_{2} \\
\text { Embedded in } \mathrm{OCT} \\
\text { compound } \\
\text { Cryosectioned } \\
10 \mu \mathrm{m} \text { thick at } \\
-20{ }^{\circ} \mathrm{C} \\
\text { Freeze-dried } 2 \mathrm{~h} \\
\left(-53{ }^{\circ} \mathrm{C} \text {, }\right. \\
0.140 \mathrm{mbar})\end{array}$ & $\begin{array}{l}\text { Soybean } \\
\text { Glycine max } \\
\text { L. } \\
\text { (Root, nodule } \\
\text { and seed) }\end{array}$ & $\begin{array}{l}\mathrm{CeO}_{2} \\
\mathrm{ZnO} \text { (Soil) }\end{array}$ \\
\hline
\end{tabular}

Table 1 (Continued)

\begin{tabular}{|c|c|c|c|}
\hline Techniques & Sample preparation & $\begin{array}{l}\text { Plant species } \\
\text { (tissue) }\end{array}$ & $\begin{array}{l}\text { NPs (cultivation } \\
\text { media) }\end{array}$ \\
\hline $\begin{array}{l}\text { SR- } \mu \text { XFM Ce } \mathrm{L}_{\mathrm{III}} \text {-edge } \\
\text { (Rico et al., 2013) }\end{array}$ & $\begin{array}{l}\text { Plunge-freezing } \\
30 \text { min in } \mathrm{LN}_{2} \\
\text { Embedded in } \mathrm{OCT} \\
\text { compound } \\
\text { Cryosectioned } \\
10 \mu \mathrm{m} \text { thick at } \\
-20^{\circ} \mathrm{C} \\
\text { Freeze-dried } 2 \mathrm{~h} \\
\left(-53{ }^{\circ} \mathrm{C},\right. \\
0.140 \mathrm{mbar})\end{array}$ & $\begin{array}{l}\text { Rice } \\
\text { Oryza sativa } \\
\text { L. } \\
\text { (Root) }\end{array}$ & $\begin{array}{l}\mathrm{CeO}_{2} \\
\text { (Hydroponics) }\end{array}$ \\
\hline $\begin{array}{l}\text { SR- } \mu \text { XFM/ } \mu \text { XAS Ti K- } \\
\text { edge (Servin et al., 2013) }\end{array}$ & $\begin{array}{l}\text { Plunge-freezing in } \\
\mathrm{LN}_{2} \\
\text { Embedded in OCT } \\
\text { compound } \\
\text { Cryosectioned } \\
40 \mu \mathrm{m} \text { thick } \\
\text { Frozen-hydrated }\end{array}$ & $\begin{array}{l}\text { Cucumber } \\
\text { Cucumis } \\
\text { sativus L. } \\
\text { (Fruit) }\end{array}$ & $\mathrm{TiO}_{2}($ Soil) \\
\hline $\begin{array}{l}\text { SR- } \mu \text { XFM Zn K-edge } \\
\text { (Wang et al., 2013) }\end{array}$ & $\begin{array}{l}\text { No sample } \\
\text { preparation, fast } \\
\text { detector MAIA }\end{array}$ & $\begin{array}{l}\text { Cowpea } \\
\text { Vigna } \\
\text { unguiculata } \\
\text { L. } \\
\text { (Root and } \\
\text { seed) }\end{array}$ & $\begin{array}{l}\mathrm{ZnO} \\
\text { (Hydroponics } \\
\text { and soil) }\end{array}$ \\
\hline $\begin{array}{l}\text { SR- } \mu \text { XFM Zn K-edge } \\
\text { (Zhao et al., 2013) }\end{array}$ & $\begin{array}{l}\text { Plunge-freezing } \\
30 \text { min in } \mathrm{LN}_{2} \\
\text { Embedded in OCT } \\
\text { compound } \\
\text { Cryosectioned } \\
30 \mu \mathrm{m} \text { thick at } \\
-20^{\circ} \mathrm{C} \\
\text { Freeze-dried } 1 \mathrm{~h} \\
\left(-53{ }^{\circ} \mathrm{C} \text {, }\right. \\
0.140 \mathrm{mbar})\end{array}$ & $\begin{array}{l}\text { Cucumber } \\
\text { Cucumis } \\
\text { sativus L. } \\
\text { (Leaf and } \\
\text { fruit) }\end{array}$ & $\begin{array}{l}\mathrm{CeO}_{2} \\
\mathrm{ZnO} \text { (Soil) }\end{array}$ \\
\hline $\begin{array}{l}\text { SR- } \mu \text { XFM/ } / \mu X A S \text { Ti K- } \\
\text { edge (Larue et al., 2014a) }\end{array}$ & $\begin{array}{l}\text { Plunge-freezing } \\
\text { (isopentane) } \\
\text { Embedded in OCT } \\
\text { compound } \\
\text { Cryosectioned } \\
20 \mu \mathrm{m} \text { thick } \\
\text { Frozen-hydrated }\end{array}$ & $\begin{array}{l}\text { Lettuce } \\
\text { Lactuca } \\
\text { sativa } \mathrm{L} \text {. } \\
\text { (Leaf) }\end{array}$ & $\mathrm{TiO}_{2}$ (Foliar) \\
\hline $\begin{array}{l}\text { SR- } \mu \text { XFM/ } \mu \text { XAS Ag } \\
\mathrm{L}_{\mathrm{III}} \text {-edge (Larue et al., } \\
2014 \mathrm{~b})\end{array}$ & $\begin{array}{l}\text { Plunge-freezing } \\
\text { (isopentane) } \\
\text { Embedded in OCT } \\
\text { compound } \\
\text { Cryosectioned } \\
20 \mu \mathrm{m} \text { thick }\end{array}$ & $\begin{array}{l}\text { Lettuce } \\
\text { Lactuca } \\
\text { sativa } \mathrm{L} \text {. } \\
\text { (Leaf) }\end{array}$ & $\mathrm{Ag}$ (Foliar) \\
\hline $\begin{array}{l}\text { SR- } \mu \text { XFM/ } \mu \text { XAS Ce } \\
\mathrm{L}_{\mathrm{III}} \text {-edge (Majumdar et } \\
\text { al., 2014) }\end{array}$ & $\begin{array}{l}\text { Frozen-hydrated } \\
\text { Plunge-freezing in } \\
\mathrm{LN}_{2} \\
\text { Embedded in OCT } \\
\text { compound } \\
\text { Cryosectioned } \\
30 \mu \mathrm{m} \\
\text { Freeze-dried } 1 \mathrm{~h} \\
\left(-53^{\circ} \mathrm{C} \text {, }\right. \\
0.140 \mathrm{mbar})\end{array}$ & $\begin{array}{l}\text { Kidney bean } \\
\text { Phaseolus } \\
\text { vulgaris L. } \\
\text { (Root) }\end{array}$ & $\begin{array}{l}\mathrm{CeO}_{2} \\
\text { (Hydroponics) }\end{array}$ \\
\hline $\begin{array}{l}\text { SR- } \mu \text { XFM Zn K-edge } \\
\text { (Zhao et al., 2014) }\end{array}$ & $\begin{array}{l}\text { Plunge-freezing } \\
30 \text { min in } \mathrm{LN}_{2} \\
\text { Embedded in OCT } \\
\text { compound } \\
\text { Cryosectioned } \\
30 \mu \mathrm{m} \text { at }-20{ }^{\circ} \mathrm{C} \\
\text { Freeze-dried } 1 \mathrm{~h} \\
\left(-53{ }^{\circ} \mathrm{C} \text {, }\right. \\
0.140 \mathrm{mbar})\end{array}$ & $\begin{array}{l}\text { Cucumber } \\
\text { Cucumis } \\
\text { sativus L. } \\
\text { (Fruit) }\end{array}$ & $\begin{array}{l}\mathrm{CeO}_{2} \\
\mathrm{ZnO} \text { (Soil) }\end{array}$ \\
\hline
\end{tabular}


Table 1 (Continued)

\begin{tabular}{|c|c|c|c|}
\hline Techniques & Sample preparation & $\begin{array}{l}\text { Plant species } \\
\text { (tissue) }\end{array}$ & $\begin{array}{l}\text { NPs (cultivation } \\
\text { media) }\end{array}$ \\
\hline $\begin{array}{l}\text { SR- } \mu \text { XFM Ce and La } \\
\mathrm{L}_{\mathrm{III}} \text {-edge (Ma et al., } \\
2015 \mathrm{~b})\end{array}$ & $\begin{array}{l}\text { Embedded in OCT } \\
\text { compound } \\
\text { Freezing in } \mathrm{LN}_{2} \\
\text { Cryosectioned } \\
40 \mu \mathrm{m} \\
\text { Air Dried?? }\end{array}$ & $\begin{array}{l}\text { Cucumber } \\
\text { Cucumis } \\
\text { sativus L. } \\
\text { (Root and } \\
\text { leaf) }\end{array}$ & $\begin{array}{l}\mathrm{CeO}_{2} \\
\mathrm{La}_{2} \mathrm{O}_{3} \\
\text { (Hydroponics) }\end{array}$ \\
\hline $\begin{array}{l}\text { SR- } \mu \text { XFM/ } \mu \text { XAS Cu K- } \\
\text { edge (Peng et al., 2015) }\end{array}$ & $\begin{array}{l}\text { Embedded in OCT } \\
\text { compound } \\
\text { Cryosectioned } \\
50 \mu \mathrm{m} \text { at }-20{ }^{\circ} \mathrm{C} \\
\text { Freeze-dried } 12 \mathrm{~h} \\
\left(-56{ }^{\circ} \mathrm{C} \text {, }\right. \\
0.280 \text { mbar })\end{array}$ & $\begin{array}{l}\text { Rice } \\
\text { Oryza sativa } \\
\text { L. } \\
\text { (Root and } \\
\text { leaf) }\end{array}$ & $\begin{array}{l}\mathrm{CuO} \\
\text { (Hydroponics) }\end{array}$ \\
\hline $\begin{array}{l}\text { SR- } \mu \text { XFM Ag K-edge and } \\
\mathrm{L}_{\mathrm{III}} \text {-edge (Stegemeier et } \\
\text { al., 2015) }\end{array}$ & $\begin{array}{l}\text { Fixed in } 3 \% \\
\text { glutaraldehyde } \\
\text { Embedded in } \\
\text { epoxy resin } \\
\text { No sectioning } \\
\text { (whole root) }\end{array}$ & $\begin{array}{l}\text { Alfalfa } \\
\text { Medicago } \\
\text { sativa } \text { L. } \\
\text { (Root) }\end{array}$ & $\begin{array}{l}\mathrm{Ag} \\
\text { (Hydroponics) }\end{array}$ \\
\hline $\begin{array}{l}\text { SR- } \mu \text { XFM Zn K-edge } \\
\text { (Zhao et al., 2015) }\end{array}$ & $\begin{array}{l}\text { Plunge-freezing } \\
30 \text { min in } \mathrm{LN}_{2} \\
\text { Embedded in } \mathrm{OCT} \\
\text { compound } \\
\text { Cryosectioned } \\
30 \mu \mathrm{m} \text { at }-20{ }^{\circ} \mathrm{C} \\
\text { Freeze-dried } 1 \mathrm{~h} \\
\left(-53{ }^{\circ} \mathrm{C} \text {, }\right. \\
0.140 \mathrm{mbar})\end{array}$ & $\begin{array}{l}\text { Maize } \\
\text { Zea mays L. } \\
\text { (Kernel) }\end{array}$ & $\begin{array}{l}\mathrm{CeO}_{2} \\
\mathrm{ZnO} \text { (Soil) }\end{array}$ \\
\hline $\begin{array}{l}\text { SR- } \mu \text { XFM/ } \mu \text { XAS (Ag } \\
\mathrm{L}_{\mathrm{III}} \text {-edge) and SR- } \\
\text { nanoXFM (Ag K-edge } \\
\text { pink beam) (Martinez- } \\
\text { Criado et al., 2015) }\end{array}$ & $\begin{array}{l}\text { Plunge-freezing } \\
\text { (isopentane) } \\
\text { Embedded in OCT } \\
\text { compound } \\
\text { Cryosectioned } \\
25 \mu \mathrm{m} \text { thick } \\
\text { Freeze-dried } \\
\text { overnight }\left(-65^{\circ} \mathrm{C} \text {, }\right. \\
0.140 \text { mbar) }\end{array}$ & $\begin{array}{l}\text { Sunflower } \\
\text { Helianthus } \\
\text { annuus L. } \\
\text { (Root) }\end{array}$ & $\begin{array}{l}\mathrm{Ag} \\
\text { Hydroponics }\end{array}$ \\
\hline $\begin{array}{l}\text { SR- } \mu \text { XFM/ } \mu \text { XAS Ce } \\
\mathrm{L}_{\mathrm{III}} \text {-edge (Hernandez- } \\
\text { Viezcas et al., 2016) }\end{array}$ & $\begin{array}{l}\text { Plunge-freezing in } \\
\mathrm{LN}_{2} \\
\text { Embedded in OCT } \\
\text { compound } \\
\text { Cryosectioned } \\
20 \mu \mathrm{m} \text { thick } \\
\text { Freeze-dried } 2 \mathrm{~h} \\
\left(-53{ }^{\circ} \mathrm{C} \text {, }\right. \\
0.140 \mathrm{mbar})\end{array}$ & $\begin{array}{l}\text { Mesquite } \\
\text { Prosopis } \\
\text { juliflora- } \\
\text { velutina } \text { s.w. } \\
\text { (Root) }\end{array}$ & $\begin{array}{l}\mathrm{CeO}_{2} \\
\text { Hydroponics }\end{array}$ \\
\hline $\begin{array}{l}\text { SR- } \mu \text { XFM/ } \mu \text { XAS Ag } \\
\mathrm{L}_{\mathrm{III}} \text {-edge and Ti K-edge } \\
\text { (Larue et al., 2016) }\end{array}$ & $\begin{array}{l}\text { Plunge-freezing } \\
\text { (isopentane) } \\
\text { Embedded in OCT } \\
\text { compound } \\
\text { Cryosectioned } \\
25 \mu \mathrm{m} \text { thick } \\
\text { Frozen-hydrated }\end{array}$ & $\begin{array}{l}\text { Lettuce } \\
\text { Lactuca } \\
\text { sativa } \text { L. } \\
\text { (Root) }\end{array}$ & 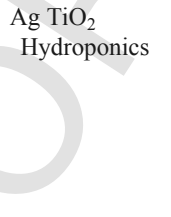 \\
\hline
\end{tabular}

\subsection{Cryofixation}

As an alternative to chemical fixation, new sample preparation protocols have been developed over the last ten years. Especially, cryofixation protocols like plunge-freezing and high pressure freezing. Cryofixation has two advantages over chemical fixation: it is achieved within milliseconds and it ensures simultaneous immobilization of all macromolecular components in their native state. Successful cryofixation is the transformation of water from a liquid to an amorphous solid state without inducing the nucleation of hexagonal ice crystals (cooling rates $>10^{5}{ }^{\circ} \mathrm{C} \mathrm{s}^{-1}$ ) which would damage morphological structures. Moreover, substances in solution (electrolytes and soluble molecules) would be excluded from the ice crystals (phase segregation), becoming more concentrated in the liquid phase surrounding the growing crystals, finally leading to elemental redistribution.

\subsubsection{Plunge-freezing}

In the plunge-freezing protocol, the sample is frozen by plunging it in a cryogen which can be propane, ethane, or isopentane cooled by liquid nitrogen $\left(\mathrm{LN}_{2}\right)$ (Vogel-Mikuš et al., 2009). The best moment to plunge the sample is when the cryogen starts being partly cloudy: this is when it is the coldest; before it becomes solid. Plunging into liquid nitrogen is not recommended as it will result in slower cooling rates, possibly leading to ice crystal formation. The sample is usually embedded in a resin (Optimal Cutting Temperature OCT resin) prior to plunge-freezing in order to facilitate subsequent cryosectioning. The OCT is a viscuous resin that does not penetrate easily into the sample, it only provides support around the sample to facilitate sample handling and cutting at cryogenic temperatures. Vogel-Mikuš et al. (2014) presents a detailed protocol that requires minimal sample handling (so less risk of damaging the tissue) and is versatile in terms of sample size. It can also be semi-automated by using automated plungers.

Plunge freezing followed by analysis under cryogenic conditions has been successfully used by Larue et al. (2014a,b, 2016) to study the fate of $\mathrm{Ag}$ and $\mathrm{TiO} 2 \mathrm{NPs}$ in both root and shoot of Lactuca sativa L., and $\mathrm{TiO}_{2}$ NPs in Cucumis sativus L. fruit by Servin et al. (2013).

It is now significantly demonstrated that ultra-rapid cryofixation over chemical fixation is the preferred strategy for stabilizing biological material close to the in vivo state (Perrin et al., 2015). The plunge freezing protocol is easy to implement giving good results for elemental mapping and speciation at the tissue and cellular level. However, since cooling rates decrease as sample size increases this protocol should be validated for large samples ( $>0.6 \mathrm{~mm}$ diameter), especially for analysis at sub-cellular level (Isaure et al., 2015).

\subsubsection{High pressure freezing}

The nucleation of ice crystals is both temperature- and pressure-dependent. High pressure $(\approx 2100$ bars $)$ synchronized with fast cooling of the sample $(\approx 20 \mathrm{~ms})$ inhibits the expansion of water and thus ice crystal formation. The high pressure freezing fixation protocol offers some advantages over plunge-freezing: automation which implies high reproducibility, and fast and homogenous freezing which allows optimal ultrastructure preservation. However, it also has some limitations: it requires the use of expensive instrumentation and it is restricted to small samples (mm range) (Lambrecht et al., 2015; McDonald, 2009).

The use of high pressure freezing has not yet been reported in our field of interest. However, it has been used for plant biology imaging studies by nano secondary ion mass spectrometry (nanoSIMS) on specific cell types or organs (glandular cells, pollen and seeds) and also on leaves (Smart et al., 2010). Some comparative studies have been performed in other research domains to assess the efficiency of such preparation protocols, for instance some researchers imaged skin samples comparing plunge freezing and high pressure freezing (Richter et al., 2007). They found that high pressure freezing gave excellent results throughout the whole skin sample, and facilitated high-resolution cellular ultrastructure visualization deep within the skin (below $10 \mu \mathrm{m}$ from skin surface) down to a resolution of about $4 \mathrm{~nm}$. For the outermost layer of skin (stratum corneum), which contains the least water, they obtained comparable results with plunge freezing and were able to achieve high sample throughput. Deeper in 
the skin, reaching more hydrated layers, a massive growth of ice crystals severely destroyed tissue organization.

\subsection{Freeze substitution}

Freeze-substitution usually follows cryofixation process. Samples are dehydrated by replacing the amorphous ice resulting from cryofixation by an organic solvent (such as acetone). This process occurs at controlled increased temperature (from liquid nitrogen temperature: $-160{ }^{\circ} \mathrm{C}$ to room temperature) to avoid melting of ice and recrystallization. This step is then followed by resin infiltration.

This protocol resulted in a well preserved ultra structure of leaf samples from the Ni hyperaccumulator Alyssum lesbiacum analyzed by nanoSIMS (Smart et al., 2010). It also proves to be better than chemical fixation in the case of the green algae Jaagiella alpicola $\mathrm{V}$. (Porta and Lopez-Iglesias, 1998). To the best of our knowledge no results are yet available on NP distribution in plants studied by SR techniques. If this protocol is efficient for ultrastructure preservation and elemental distribution, embedding of the sample in resin is not recommended for speciation analyses. The embedding protocols involve several steps such as fixation, dehydration, penetration, and polymerization where the speciation of elements and its distribution might be modified, and samples may be contaminated.

\section{4. (Cryo)sectioning}

Sample sectioning can be done under cryogenic conditions for cryofixed samples or at room temperature for resin embedded samples (either chemically fixed or after freeze-substitution). One of the main issues with sectioning is the loss of tissue ultra structure due to knife marks and sample compression/distortion. This previously mentioned problem is of particular importance for cryosectioning plant samples that are composed of different density materials alternating dense and rigid cellulose cell walls, fragile cytoplasms, and gas cavities. Over the last two decades, significant efforts to minimize the deleterious artifacts associated with cryo-sections (e.g. sharper knife edges or vibrating knives) have yielded only limited successes (Richter et al., 2007).

For frozen-hydrated samples, the cryo-microtome set-up needs to be optimized for each tissue type: chamber temperature (usually around $-20^{\circ} \mathrm{C}$ ), sample cold finger and knife temperatures, type of knife (e.g. diamond, stainless steel, or tungsten), cutting velocity, and section thickness (Vogel-Mikuš et al., 2009). The typical thickness used for SR- $\mu$ XFM analysis is from 10 to $40 \mu \mathrm{m}$. For SR-nanoXFM applications $0.5-5 \mu \mathrm{m}$ thickness will give good results. Quality assessment of the cryo-sections is important to avoid losing time removing and transferring another sample (particularly for in vacuum experiments this is time consuming). A good option is to have a binocular or a microscope mounted on the cryomicrotome to check the quality of the section and to verify that mounting on the sample holder did not lead to damages before analysis.

A remark often made in the case of NP studies is the possibility to displace them within a sample or cross contaminate samples when using non-disposable knives. Samples must be rinsed before freezing or inclusion with either ultrapure water or $0.01 \mathrm{M} \mathrm{HNO}_{3}$ to remove at least the loosely bound NPs from the surface of the samples. The cutting knife should be clean after each section, a knife dedicated to each sample is recommended when using disposable knifes. After sectioning, the sample is usually placed between two polymer foils (e.g. Ultralene (SPEX SamplePrep), Pioloform, or Mylar) and mounted on the sample holder. For cryo-sections this should be done always under cryogenic conditions using pre-cooled material and tools.

\subsection{Freeze-drying}

If the beamline does not have a cryogenic stage (or a fast detector), cryofixed samples need to be dehydrated. Typically this is achieved by freeze-drying. This is a sensitive step because plant samples usually contain a high percentage of water. If not properly done it can lead to elemental redistribution, loss of elements, shrinking of the specimens and severe morphology distortion. To obtain optimal results, it is essential to keep the samples always frozen during transfer from plunger device to cryotome and then to freeze drier. Freeze-drying should be done at the lowest temperature possible, under low pressure $\left(\approx 10^{-2}\right.$ mbar). In particular, samples should be brought back to room temperature and pressure gradually to prevent shrinking of the specimens. Flatness of the sample is another issue; it can be maintained by placing samples between two rigid layers (e.g. Al or $\mathrm{Cu}$ disks).

Freeze-drying is the most frequently used sample preparation protocol for mapping and speciation of NPs in plants. In general, the freeze-drying protocol has provided enough sample preservation to enable distinction of different tissue layers (e.g. epidermis, casparian strip, vascular bundles) and cell structures. However, the main problem detected was the presence of some empty cells. The content could have been lost during sample preparation; probably due to low cooling rates and/or defrosting of the sample at some stage of the protocol or during sectioning. Freeze-drying seems to be a good alternative for studies at the tissue level, and for studies at the cellular level following careful protocols. However, it might not be the best option for experiments at the subcellular level using SR-nanoXFM. Whenever possible, the use of (frozen)-hydrated specimens is the preferred option (Donner et al., 2013).

Some tests have been performed to assess the suitability of freeze-drying for elemental distribution experiments. In leaves of freeze-dried Senecio anomalochrous H., elemental distribution was similar to that of frozen-hydrated samples, however concentrations in the freeze-dried samples were higher by a factor of 3 (Tylko et al., 2007). Further analyses demonstrated that this discrepancy was linked to the loss of oxygen under the beam during the first minutes of analysis (by $\mu$ PIXE). Vogel-Mikus et al. (2009) obtained very good results using this technique in terms of tissue preservation in root samples of Viola lutea analyzed by $\mu$ PIXE. In another study, authors observed altered elemental distribution at the cellular level for some elements (especially $\mathrm{K}, \mathrm{P}$, and $\mathrm{Ca}$ ) in the leaves of Thlaspi praecox (Vavpetic et al., 2015).

Freeze-drying has also been found to be suitable for speciation analysis. Isaure et al. (2006) reported no changes in Cd speciation when comparing freeze-dried and frozen hydrated roots and trichomes of Arabidopsis thaliana L. seedlings. However, (Sarret et al., 2009) reported slightly different results for $\mathrm{Zn}$ speciation between the frozen hydrated and the freeze dried state in Arabidopsis halleri L.. Likewise, in a study comparing the speciation of $\mathrm{Ag}$ in the leaves of $\mathrm{Vi}$ gna unguiculata L. and Triticum aestivum L. freeze-dried vs. frozen hydrated, authors suspected that the freeze-drying step induced sulfidation of $\mathrm{Ag}$ (Wang et al., 2015). This kind of comparison is still very limited across different elements, plant species and tissues. More studies are needed, in particular to investigate the impact of freeze-drying on NP speciation. Since it is difficult to affirm if freeze drying may or may not alter the speciation of the element of interest, when possible, it is recommended to work on frozen hydrated samples. 


\subsection{Critical point drying}

During freeze-drying processes, shrinkage and distortion can occur - especially in highly hydrated samples - because of the surface tension created at the gas-solid junction during sublimation. This can be avoided using critical point drying. This protocol is based on the existence of a critical point in which there is no apparent difference between gas and liquid state, the surface tension reduces to zero. The critical point of water is not convenient (too high temperature $374{ }^{\circ} \mathrm{C}$, and pressure 220 bar), so in this protocol water is replaced by another transitional medium whose critical temperature for an attainable pressure is just above ambient: $\mathrm{CO}_{2}$ (critical point $31^{\circ} \mathrm{C}, 73.8$ bar). However, an intermediate step is needed because $\mathrm{CO}_{2}$ and water are not miscible. This step is the replacement of water by acetone (or methanol, ethanol, amyl acetate) which in turn is replaced by high pressure liquid $\mathrm{CO}_{2}$ before increasing the temperature above the critical point. Liquid $\mathrm{CO}_{2}$ changes to vapor without changing density eliminating the surface tension effects. Pressure can then be gradually released, allowing the gas to escape and leaving a dried sample.

Critical point drying has been applied mostly for X-ray tomography and scanning electron microscopy analyses on whole organ samples. It was used to study the roots of Asplenium theciferum M (Leroux et al., 2009). and more recently for very fragile arthropod nervous system samples (Sombke et al., 2015). It allowed localizing carbon nanotubes in Cucumis sativus L. roots (Cañas et al., 2008), silica microparticles in the leaves of Phragmites australis Cav. (Schaller et al., 2013), and of $\mathrm{TiO}_{2}$ NPs adhered on leaves of Lemna minor L. (Li et al., 2013). However, this protocol does not allow the preservation of ultra structure, and due to the water exchange step (use of acetone, methanol, or ethanol) is not recommended for XAS experiments.

\subsection{Cryo-sample handling}

The most sensitive steps during sample preparation and transport of cryogenic samples are: (i) Recovery of plant cross-sections: frozen sections are always difficult to attach to sample holder or support (e.g $\mathrm{Si}_{3} \mathrm{~N}_{4}$, Ultralene, or Mylar). Thaw mounting on the support for attachment is tempting but this will most likely lead to artifacts (e.g. elemental redistribution), even if this process is only thawing the sample for a few seconds. (ii) Mounting on adequate holder for analysis or freeze drying. (iii) Transfer from the cryomicrotome to the analysis chamber/freeze-dryer, this needs to be done carefully under $\mathrm{LN}_{2}$ or $\mathrm{LN}_{2}$ vapours to avoid melting and possible ice formation on the sample surface. (iv) Sample transportation. Some beamlines are equipped with a sample preparation lab which offers more flexibility during the experiment and less sample handling (but more work on site). If not available, samples are prepared at the home institution and then shipped to the beamline. Dry ice shipping is a possible option for sample transportation, but a safer and optimal alternative is shipping samples in specialized liquid nitrogen vapor dewars that can preserve samples for several days and are authorized by air transport regulation.

\section{Data acquisition}

A beamline for SR-XFM and XAS analyses is usually composed of a monochromator, various optical devices for focusing and collimating the beam, a diagnostic of the incident beam, a motorized sample stage, an XRF detector, and an optical microscope to visualize the sample. As an example Fig. 4 displays the sketch of the ID21 beamline at the ESRF, where many of the following examples were acquired.It is not the aim of this review to cover details of beamline instrumentation; the reader can find more information in recent revisions (Sarret et al., 2013; Pushie et al., 2014; Grafe et al., 2014).

XRF detector

(SDD)

\section{))}

$\longrightarrow \mathrm{LN}_{2}$

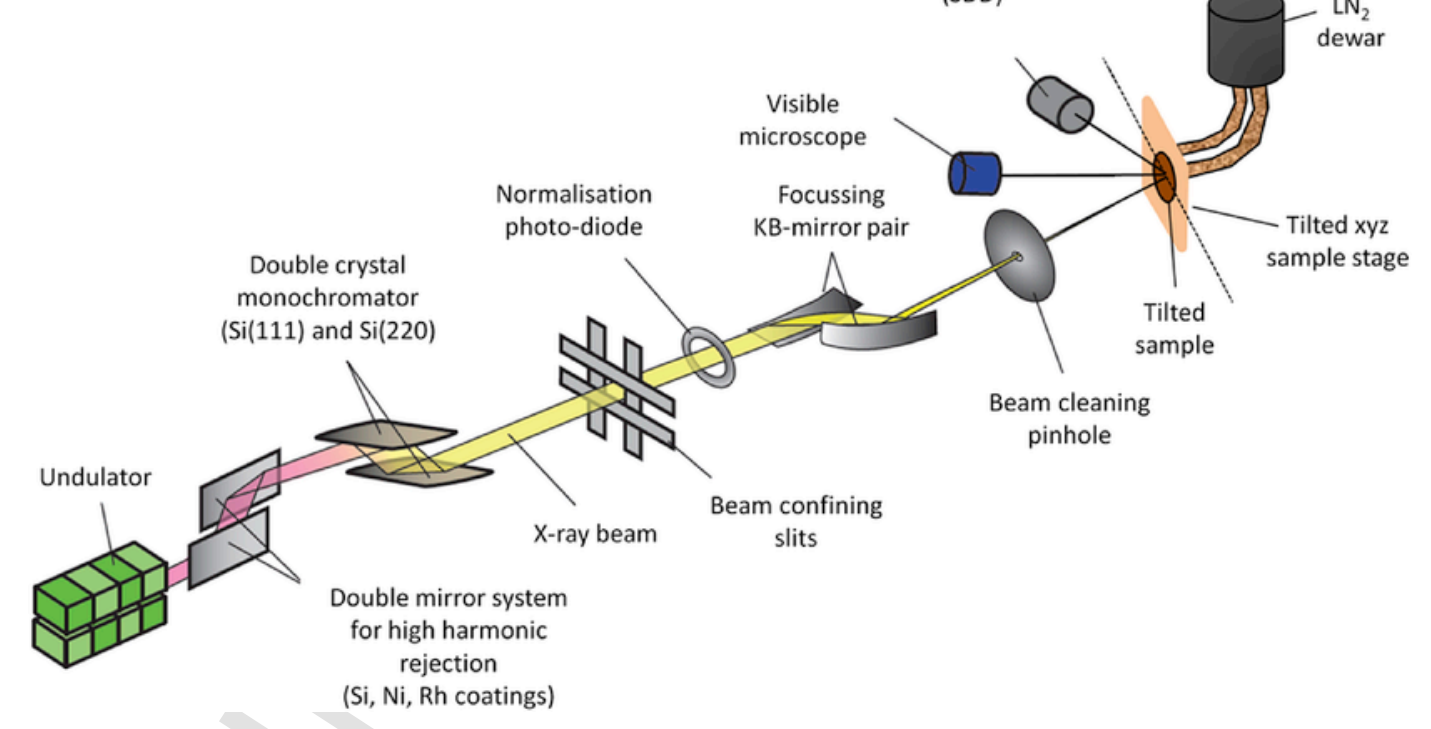

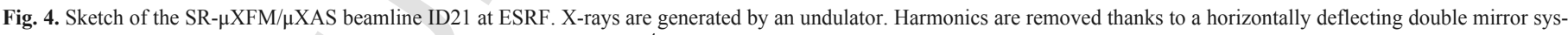

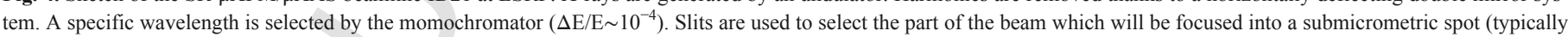

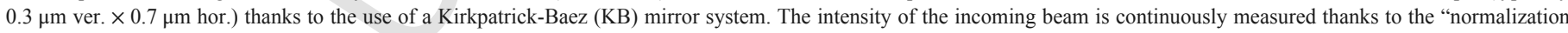

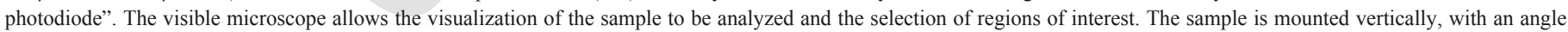

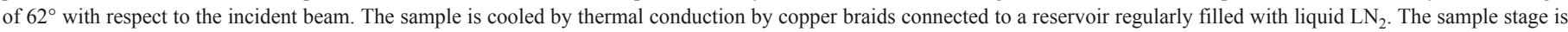
raster scanned horizontally and vertically. The XRF emitted by the sample is recorded by a Silicon Drift diode (SDD) detector. 


\subsection{Sample environment}

Systems for sample cooling during data acquisition are of high interest for the analysis of plant samples because they allow to study frozen hydrated samples (see sample preparation section). Moreover, they limit possible radiation damage effects. Various cooling systems are available depending on the beamlines. Cryogenic stages based on a Peltier stage exist for sample analysis down to $-30{ }^{\circ} \mathrm{C}$. Alternatively, other systems use conductive cooling from a $\mathrm{LN}_{2}$ filled dewar connected to the sample holder by copper braids. The advantage of this system is the very low temperature $\left(\approx-150{ }^{\circ} \mathrm{C}\right)$ and the absence of vibrations (Salome et al., 2013). Recently, a system that allows the combination of $\mu$ XFM and TEM has been described in Fakra et al. (2015). Cryojets, which are used for X-ray protein crystallography, are not well adapted for larger samples and longer acquisition times because they generate vibrations.

\subsection{Micro and nano SR-XFM}

Two types of beamlines (tender and hard X-ray) can be used to study ENMs in plant samples. Tender X-ray (energy from 2 to $8 \mathrm{keV}$ ) beamlines can be used to study low $\mathrm{Z}$ element $(\leq \mathrm{Fe})$ containing ENMs such as $\mathrm{TiO}_{2}$ (Ti K-edge $\left.4.966 \mathrm{keV}\right), \mathrm{Ag}\left(\mathrm{Ag} \mathrm{L} \mathrm{L}_{\mathrm{III}}\right.$-edge $\left.3.351 \mathrm{keV}\right)$, CdSe (Cd L $\mathrm{III}^{-}$-edge $3.538 \mathrm{keV}$ ), lanthanide oxides (e.g. Ce and $\mathrm{La}$ $\mathrm{L}_{\mathrm{III}}$-edge) and Fe-containing (Fe K-edge $7.112 \mathrm{keV}$ ) NPs. Hard X-ray (energy $>8 \mathrm{keV}$ ) beamlines can be used to study heavier elements $\left(>\mathrm{Fe}\right.$ ) (e.g. $\mathrm{ZnO}, \mathrm{CuO}, \mathrm{CeO}_{2}, \mathrm{Ag}, \mathrm{CdSe}, \mathrm{CdZnS}$ ) in most cases using the K-edge transition. The XRF yield of $\mathrm{K}$ shell is about ten times higher than average $\mathrm{L}$ shell for $\mathrm{Ag}$ and $\mathrm{Cd}$, and about five times higher for heavier elements such as $\mathrm{Pb}, \mathrm{Hg}$ and $\mathrm{Au}$. Thus, for a given photon flux, a better detection limit may be obtained using high energy beamlines and K-edge excitations (X-ray data booklet, http:// xdb.lbl.gov/). An option to increase the flux $(\approx \times 100)$ is the use of "pink" beam $(\Delta \mathrm{E} / \mathrm{E} \sim 1 \%)$ instead of monochromatic $(\Delta \mathrm{E} / \mathrm{E} \sim 0.1 \%)$ (Martinez-Criado et al., 2015). The disadvantage is that this mode cannot be coupled with XAS. A great interest of working in the tender $\mathrm{X}$-ray domain is the possibility to record the signal of light elements $(\mathrm{P}, \mathrm{S}, \mathrm{Cl}, \mathrm{K}, \mathrm{Ca})$ in addition to the signal of the element of interest. These elements provide an image of the tissues and cells. Imaging $\mathrm{P}$, $\mathrm{S}$ and $\mathrm{Cl}$ generally is not possible on hard X-ray beamlines because detectors sensitive to hard X-rays are poorly sensitive to soft X-rays, and because measurements are often done in air, which attenuates the XRF of light elements. $\mathrm{K}$ and $\mathrm{Ca}$ still may be measured, and transition metals such as $\mathrm{Zn}$ and $\mathrm{Cu}$ may also provide a good representation of plant structures. The beam size on SR- $\mu \mathrm{XFM} / \mu \mathrm{XAS}$ beamlines ranges from a few $\mu \mathrm{m}$ to a few hundred $\mathrm{nm}$. This resolution is sufficient to image the various tissues and plant cells, but identifying sub-cellular structures is difficult. Some beamlines now offer a nanofocused beam down to a few tens of nm. A few of them couple nanoXFM and nanoXAS (Martinez-Criado et al., 2015), and their number will probably increase. Imaging subcellular structures becomes possible, but the sample preparation needs to be optimal to preserve these structures.

Before launching a SR-XFM acquisition, the sample to detector distance and/or the incident flux should be optimized to keep detector deadtime in the linear range for appropriate correction $(\leq 20-30 \%)$. The deadtime of an XRF detector is the time-lapse right after the counting of a photon, during which the detector processes the information and is not able to register a new photon. This step is important in the case of plant tissues containing NPs since the presence of aggregates would increase the local concentration of an element, possibly leading to high detector deadtime. SR-XFM acquisition is done by raster scanning the sample under the beam using two common schemes: step-by-step (the slowest option) and continuous (on-the-fly) acquisition mode in one direction (Pushie et al., 2014). This latter option is faster and avoids the risk of missing a particle if the steps are large (as typically done for finding regions of interest (ROI) in samples), since the signal is recorded continuously, and then split into pixels. Appropriate scanning step size is important for resolution, a 50\% overlap (the step size is half of the beam size) is generally considered as a good option for mapping. Fig. 5 illustrates the case for particles of different sizes scanned with $0-50 \%$ overlap. With $0 \%$ overlap, the circles in Fig. 5A are poorly resolved and the same object can be imaged differently, or objects of different sizes can give the same image, depending on the position of the beam relative to the particle (Fig. 5A-D). With 50\% overlap, there is less impact of the beam position, and the chance of missing particles is minimal (Fig. 5E-H). However, objects of comparable size to the beam are not correctly resolved (Fig. 5F and G). A size two-fold larger than the beam is still insufficient to correctly image the object's morphology (Fig. 5H). With a particle four-fold larger than the beam, one can have a rough image of its shape (Fig. 5I and J). Another interesting point to note is that a "corona" or halo of one to two pixels wide having lower intensity, as observed in Fig. 5H, should not be blindly interpreted as zone of NPs dissolution or formation of secondary phase. This corona might be the result from the beam hitting partly the particle and the matrix.

In addition to scanning strategy, the data recording strategy is equally important. Some SR-XFM beamlines propose a XRF-ROI mode, in which each pixel of the map contained XRF intensities measured in predefined ROIs, typically a few tens of eV around the $\mathrm{K} \alpha$ or $\mathrm{L} \alpha$ fluorescence emission lines of the elements of interest. Presently, most beamlines offer a hyperspectral acquisition mode, which means that each pixel contains a full XRF spectrum. This mode is by far preferable to the ROI mode for several reasons. First, it allows the extraction of the XRF spectrum from selected zones of the map. Averaging the XRF signal from several pixels in a region may help to better evidence the presence of elements at low concentrations. Second, this mode allows accurate data treatment such as peak deconvolution, quantification, and statistical analyses (see data analysis section).

\subsection{Micro and nano XAS}

Spatially resolved XAS is a very useful complement to SR-XFM since it allows determining the speciation of a chemical element at micro and nano-scale in a plant sample. XAS is divided in two regions according to the information that can be obtained, X-ray absorption near edge structure (XANES) and extended X-ray absorption fine structure (EXAFS) (Grafe et al., 2014). Spatially resolved XAS is most often applied in the XANES region. Here we describe different XANES acquisition modes, major pitfalls of the technique, and possible ways to overcome them. This review will focus only on XRF acquisition modes. XAS imaging techniques in transmission mode exist, like the fast $\mu$ XANES mapping (Muñoz et al., 2006), full-field $\mu$ XANES (Fayard et al., 2013), and nanoscale full field soft X-ray microscopy (Guttmann et al., 2012; Bittencourt et al., 2013) but applications are restricted to concentrated samples $(>1 \%$ content of the element of interest).

The most common acquisition mode is the single point. Once elemental distribution maps have been obtained by SR-XFM, the beam is re-positioned on a selected spot of the map, and a scan in energy allows recording the XANES spectrum of the element of interest. Fast energy scans with continuous movement of the monochromator and on-the-fly data recording are preferred to step-by-step acquisition mode. This is advantageous for biological samples which are more 

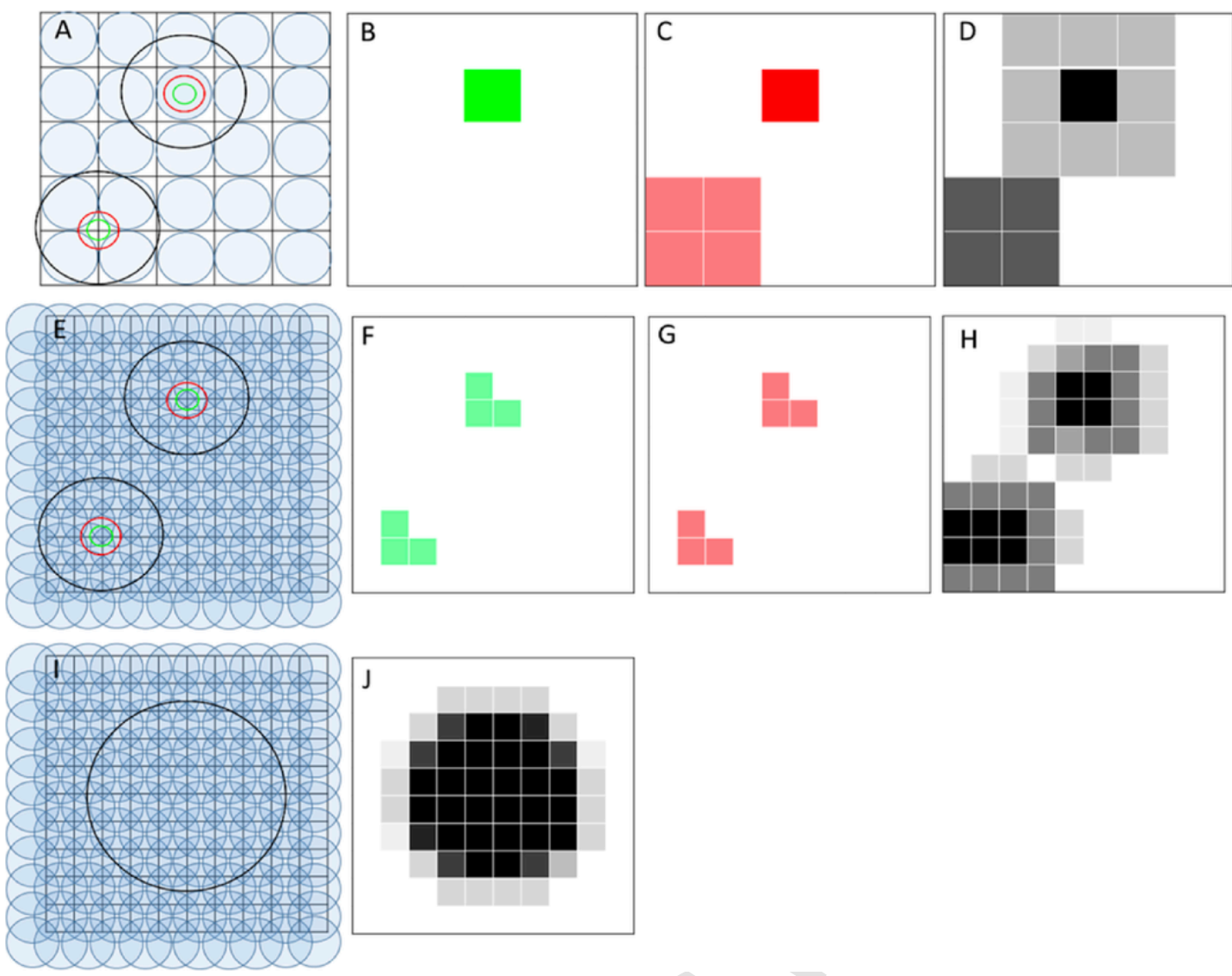

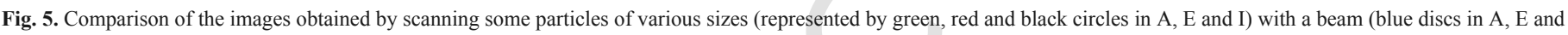

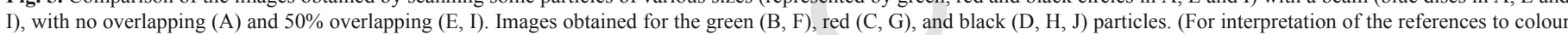
in this figure legend, the reader is referred to the web version of this article.)

sensitive to radiation damage effects. Thus, it is better to record a series of fast energy scans $(<1 \mathrm{~min})$ and compare them before averaging for final data analysis (see (Castillo-Michel et al., 2016) for examples on S K-edge XANES). In addition, for spots or regions of interest of similar composition, the sample can be moved between scans to limit radiation damage. Data acquisition under cryogenic conditions is preferable, both to limit radiation damage and also to improve the quality and amplitude of the signal by decreasing the thermal disorder in the material. The single point acquisition mode has an important limitation; it may give distorted spectra when the target object is comparable in size or slightly larger than the beam. During the energy scan, beam drifts induced by mechanical instabilities are observed, so the beam moves on-off the particle. As a result, the XANES spectrum is distorted and in most cases not suitable for interpretation (Fig. 6A and B). To overcome this problem, the alternative of full XANES mapping has been developed at some beamlines. It consists in recording the same map at each energy step of the XANES spectrum. The stack of maps (Fig. 6C) is then aligned to correct both beam and sample drifts. Then, XANES spectra can be extracted from this stack of images on a selected pixel or zone of the map. This approach not only allows obtaining good quality spectra on spots having a size comparable to the beam, but it also allows enhancing the statistics for diluted regions, by integrating the signal on several pixels. And last but not least, it allows extracting spectra in any zone of the map, and obtaining the speciation in 2D. The drawback of this mode is the long acquisition time; developments in detector technology and data analysis strategies are rendering this method more and more applicable (Grafe et al., 2014). Some metals or metalloids present different redox states associated with significant changes in the XANES features (position of the absorption edge, presence of an intense pre-edge). In that case, recording the $\mu$ XRF maps at few energies (2-6) may be sufficient to map the distribution of the element under its various oxidation states (e.g (Pickering et al., 2006).).

\section{Data analysis}

The success of the experiment will also depend on careful data analysis and interpretation. In this section, the main aspects of SR-XFM and XAS data analysis are illustrated with examples. A summary of the main steps involved on data analysis for SR-XFM and $\mathrm{XAS}$ is presented in Fig. 7.

\subsection{SR-XFM data analysis}

In this section, the analysis of SR-XFM hyperspectral data, where each pixel of the map contains a full XRF spectrum will be presented. As explained in data acquisition section, this is the most common and preferable acquisition mode. The first step of the analysis is the normalization of the data by the incident intensity (I0), normalization by dwell time, and detector dead time correction. Interpretation of the data from pixels affected by high detector deadtime should rest at a qualitative level. 

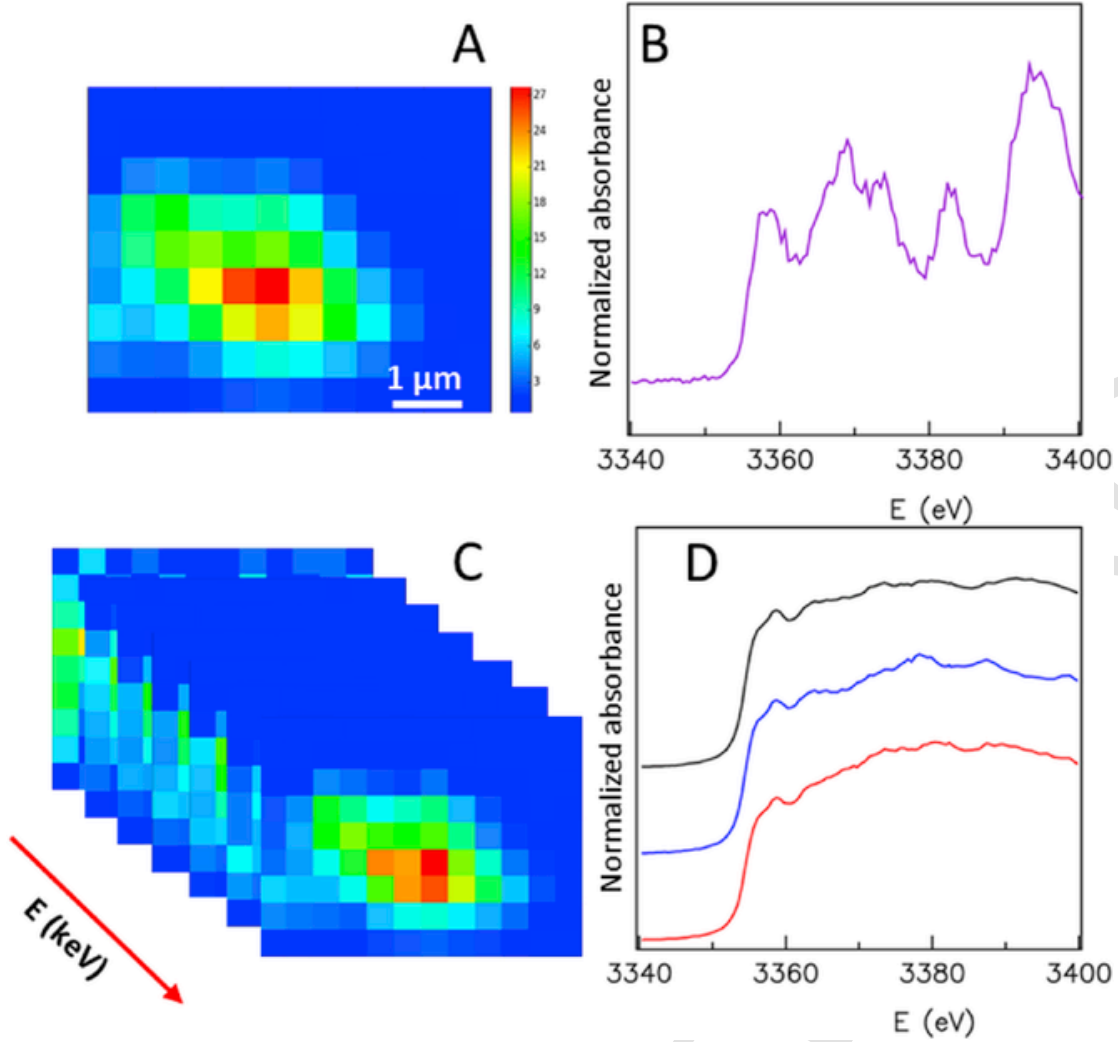

Fig. 6. Comparison of data obtained by single point and full-XANES mapping acquisition modes. A) Temperature $\mu$ XRF map of the spot analyzed (red is the most intense and blue the least in the colorbar) and B) single point $\mu$ XANES spectrum showing distortions due to drifts during the energy scan. C) Stack of $\mu$ XRF maps obtained at each energy step and D) $\mu$ XANES spectra obtained from the sum of all pixels is in black, $\mu$ XANES from the most intense pixels (red) is in red and $\mu$ XANES from the diffused areas (green) is in blue. (For interpretation of the references to colour in this figure legend, the reader is referred to the web version of this article.)

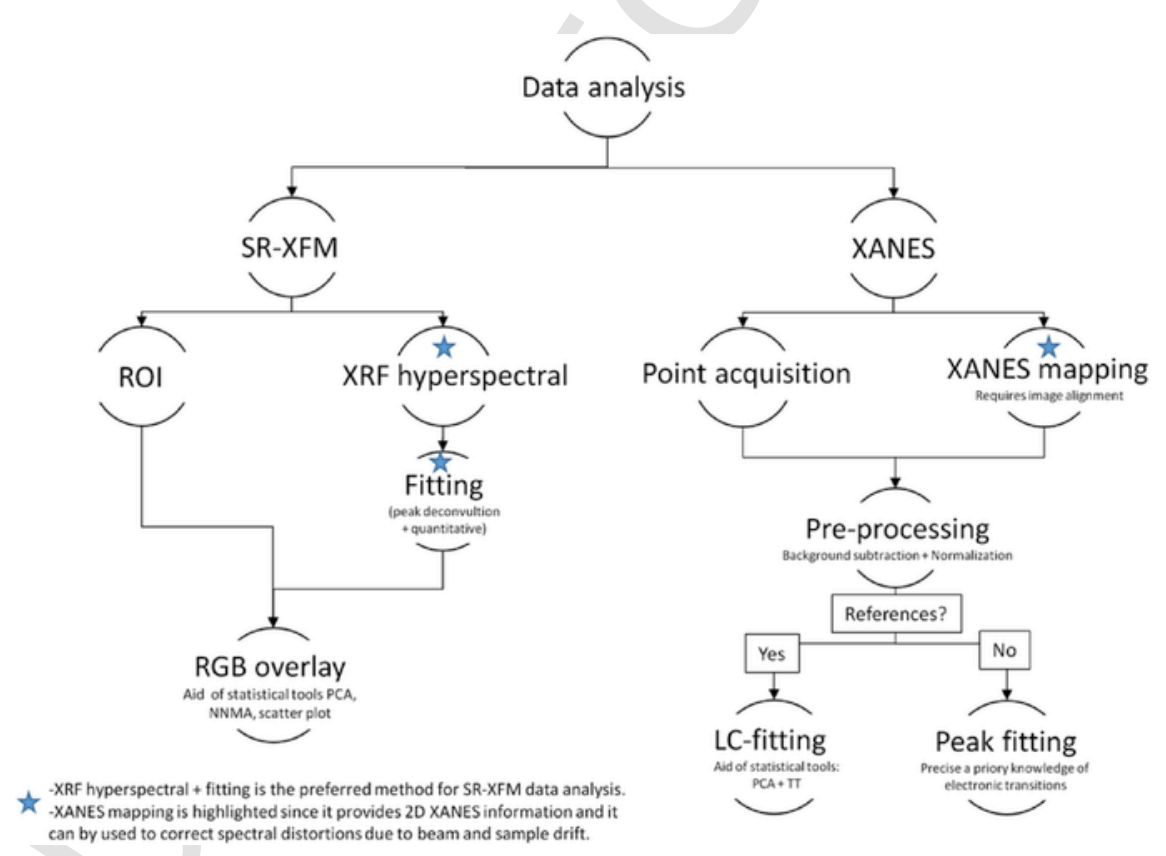

Fig. 7. Scheme presenting the main steps in the process of data analysis for SR-XFM and XANES.

An example of step-by-step procedure for SR- $\mu$ XFM data analysis in a root cross section of wheat (Triticum aestivum $\mathrm{L}$.) exposed to $\mathrm{Ag}_{2} \mathrm{~S}$ NPs is given in Fig. 8. Fig. 8A displays the image corresponding to the XRF emitted by all the elements in the sample. In Fig. 8B the sum XRF spectrum from all pixels in the scanned region is displayed. The simplest analysis of these data consists in attributing each XRF peak to an element based on tabulated X-ray emission lines and defining ROIs. Dedicated XRF data analysis programs al- 

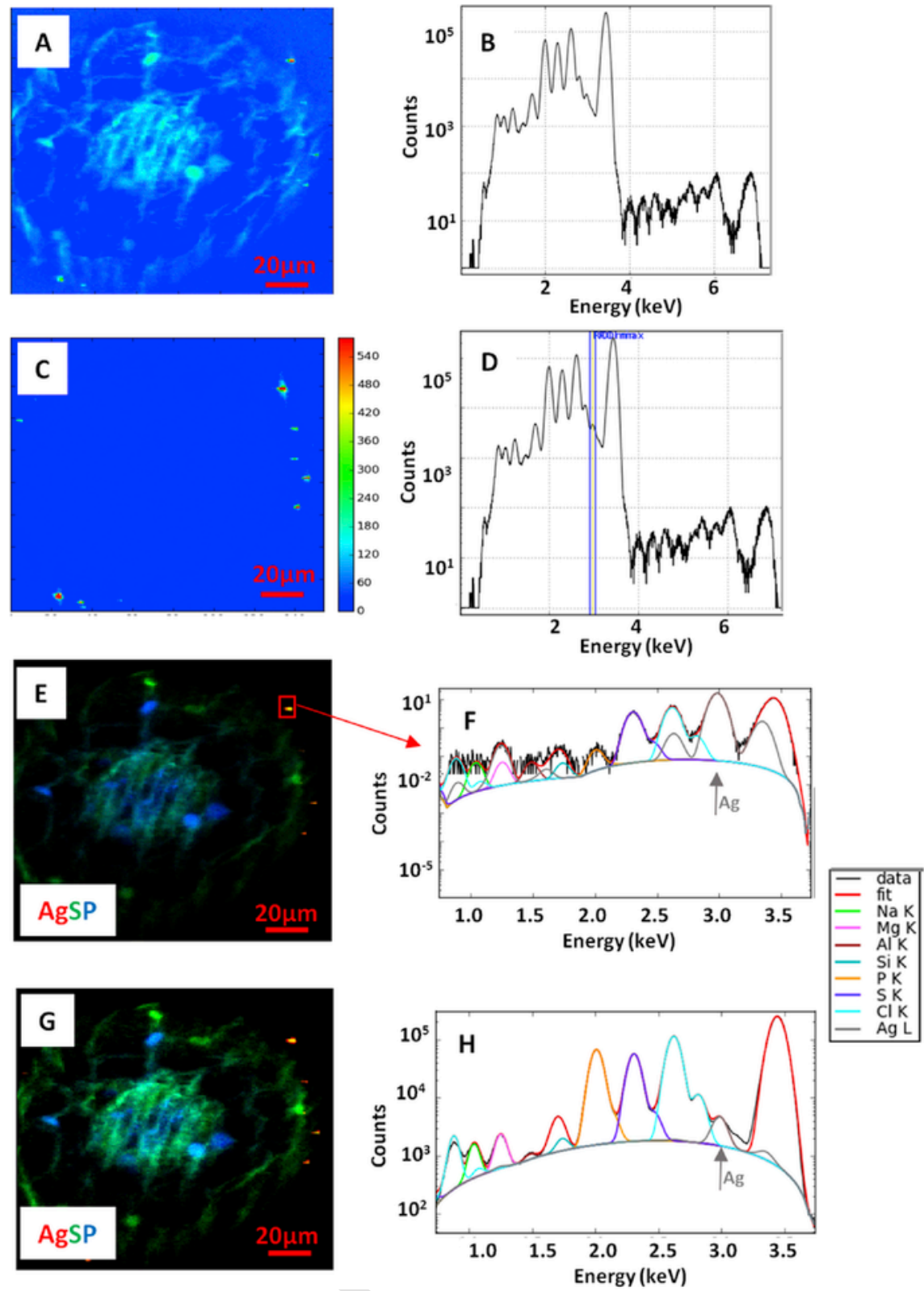

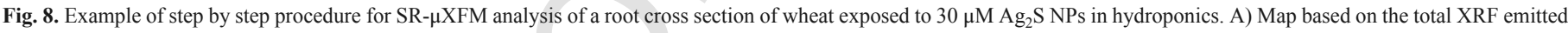

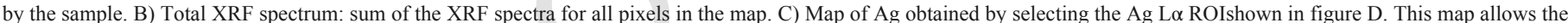

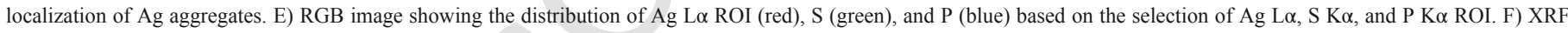

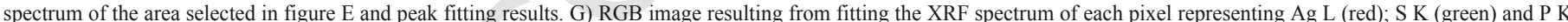

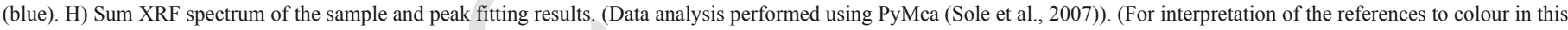
figure legend, the reader is referred to the web version of this article.)

low easy peak identification based on theoretical XRF emission lines. The relative intensity distribution of an element is visualized by defining a ROI around characteristic element emission lines (e.g. $\mathrm{S} \mathrm{K} \alpha$ ). For example, in Fig. $8 \mathrm{C}$ the distribution of Ag aggregates in the sample is shown by the selection of the Ag L $\alpha$ ROI (Fig. 8D). Most image analysis software allow the simultaneous visualization of 3 elements in false color which is very useful for the localization of NPs in complex matrices such as plant tissues and for selection of areas of interest for higher resolution SR-XFM or XAS analyses. Fig. 8E shows the image of the sample corresponding to $\mathrm{Ag} \operatorname{L} \alpha$ in red, $\mathrm{S} \mathrm{K} \alpha$ in green, and $\mathrm{P} \mathrm{K} \alpha$ in blue.
This quick analysis provides rough insights on elemental localizations and associations, and is very useful for data visualization during acquisition. In most of the cases, the $\mathrm{K}$ emission lines of metals are sufficiently distinct in energy so that the ROI approach is free of interferences. However, some interference could be found, i.e, for the $\mathrm{K}$ lines of $\mathrm{Ca}$ and $\mathrm{K}, \mathrm{Fe}$ and $\mathrm{Mn}$ or $\mathrm{Cu}$ and $\mathrm{Zn}$. In the case of $\mathrm{L}$ emission lines, there could be some interference with the $\mathrm{K}$ lines of lighter elements (e.g., $\mathrm{K} \mathrm{K} \alpha$ and $\mathrm{Cd} \operatorname{L} \alpha$, or $\mathrm{Ag} \operatorname{L} \alpha$ and $\mathrm{Ar} \mathrm{K} \alpha$ ). In this case, peak deconvolution is useful. In addition, possible pile up and escape peaks should be tested, and background subtraction is necessary. Further information on pile-up and escape peaks could be found in Pushie et al. (2014). These options are implemented in most of XRF 
data analysis packages (e.g. non-linear least square fitting approach for PyMca (Sole et al., 2007) and GeoPIXE (Ryan et al., 2005). Once these operations have been done, a set of "clean" maps (fitted images) for each element are obtained and can be used for unambiguous interpretation (Fig. 8G). Since the pixel by pixel XRF data is still preserved it is possible to come back and test if the fitting procedure was correct. It is also quite useful to extract full XRF spectra from different selected areas to compare elemental ratios qualitatively or for quantification (see below). This option is of special interest in the study of NPs in plant samples, as they often contribute in a very low proportion to the sum XRF spectrum of the sample. For example, the XRF spectrum of a single Ag NP aggregate (Fig. 8F) shows the higher relative intensity coming from Ag compared to the full map XRF sum spectrum (Fig. 8H). Inspecting sum spectra from regions or pixels is useful to avoid misinterpretations coming from overlapping elemental signals or sample preparation artifacts (e.g. scattering from ice crystals).

\subsubsection{SR-XFM multivariate data analysis}

As shown above, the simple comparison of the elemental maps (ROI-based images or fitted images) can provide insights on elemental correlations and chemical associations. To go further in the analysis, statistical approaches can be used.

Principal Component Analysis (PCA) is a multivariate analysis method that identifies the lower number of independent uncorrelated variables (Principal Components) needed to explain the maximum variance of a large set of data. In image analysis, this procedure helps to recognize patterns and to compress the data. Results can be visualized as either eigenimages or eigenvectors. Based on the number of components obtained, Non Negative Matrix Approximation (NNMA) could also be applied to obtain the corresponding images and vectors. Both PCA and NNMA methods reduce the number of components of the image to help in their interpretation. The difference between them is that, while PCA provides images with positive and negative values, NNMA only produce images with positive values (Lee and Seung, 1999). Hence, the visualization of the PCA components is more useful to study the correlation of the different elements of the sample, i.e to study the preferential association of NPs with some elements in plant tissues. In the example of our root cross section, Fig. 9A shows the RGB image that represents the three main components that explain $95.45 \%$ of the variance of the data set and the corresponding eigenvectors. PC1 (blue) is the main component $(64.9 \%)$ with high contributions from elements of plant structure $(\mathrm{P}, \mathrm{S}$, and $\mathrm{Cl}$ ), $\mathrm{PC} 2$ (green, $16.60 \%$ ) with contributions from $\mathrm{S}$ and $\mathrm{Cl}$, and $\mathrm{PC} 3$ (red, $13.95 \%$ ) is mainly Ag. In this image we can see the co-localization of Ag and S. On the other hand, NNMA would help in the visualization of disperse elements such as the small particle aggregates in plant roots. In Fig. 9B, NNMA components are also shown in RGB, we can see that this visualization increases the contrast of the PC3 components allowing an easier localization of the Ag NPs (in red). In this case from PC2 (green) it can be observed that $\mathrm{Cl}$ is a trace element in the OCT resin used to embed the sample. $\mathrm{S}$ is also included in this component so the information about $\mathrm{Ag}-\mathrm{S}$ co-localization is lost. It is important to note that these statistical procedures are not always required. For example, the RGB image representing Ag, $\mathrm{P}$, and $\mathrm{S}$ after image fitting (Fig. 8G) already shows the interesting patterns (co-localization of $\mathrm{Ag}$ and $\mathrm{S}$, and tissue structure). It is also possible to perform PCA and NNMA on full XRF spectrum data as shown in Fig. 10. In this example Lactuca sativa L. plants exposed to Ag NPs by foliar deposition are analyzed at $7.2 \mathrm{keV}$ to obtain $\mathrm{Fe}, \mathrm{Ca}, \mathrm{K}, \mathrm{Ag}, \mathrm{Cl}, \mathrm{S}$ and $\mathrm{P}$ distribution maps. In this case, NNMA allowed the visualization of all elements with 2 components, in which PC3 is presenting the co-localization of $\mathrm{Ag} \mathrm{NP}$ aggregate with Fe.
It is also informative to draw scatter plots of XRF intensities between elements (Fig. 9C), and to calculate Pearson correlations between the different elements of the sample. Another interesting option is image operations, such as subtraction of maps, which can give a direct evaluation of the correlation or anticorrelation between elements. Some or all of the above mentioned options are implemented in XRF data analysis packages such as PyMca, GeoPIXE, or MAPS. SR-XFM data could also be analyzed using common statistical packages through the exportation of XRF spectra from the maps. In this case, a set of XRF maps (as opposed to a single map) can be analyzed. This approach was used recently by Larue et al. (2016) on a set of XRF spectra obtained by $\mu$ PIXE, and allowed to test the influence of the exposure to $\mathrm{TiO}_{2}$ and Ag NPs on the homoeostasis of various elements, using PCA and linear discriminant analysis. Examples for the use of multivariate analysis methods (e.g. factor and cluster analysis, matrix factorization, discrimination analysis, etc) in the treatment of spatially resolved SR-XFM data are still scarce. These methods have been recently applied for the analysis of data from wheat grains (Singh et al., 2014), rock samples (Osán et al., 2014) and human tissues (Chwiej, 2010) but, to our knowledge, there are no reports concerning plants/ ENMs interactions.

\subsubsection{SR-XFM quantitative analysis}

Quantification of elemental abundance also can be achieved on the basis of XRF intensity on a per-weight or per-area basis (often neglecting thickness). Main steps to perform this quantification include: i) instrument calibration, ii) measurement of the intensity of the peaks of the element of interest, iii) deadtime correction, iv) background correction, and v) matrix correction (if necessary). Additional information could be found in (Rousseau, 2001; Pushie et al., 2014; West et al., 2015). The calibration procedure is done by relating the measured fluorescence peaks to the peak obtained by measuring a standard of known composition, having a matrix similar to the sample (organic matrix in the case of plants). However, available methods for quantification have some inherent uncertainty derived from the accumulation of all random and systematic errors produced during the analysis: sample preparation, measurement of both peak and background intensities, slope of the calibration line and corrections for matrix effects (Rousseau, 2001). This uncertainty is greater for the quantification of NPs in plant tissues using SR-XFM and derives mainly from the fact that NPs are present in plant tissues in the form of aggregates of different sizes and/or as ionic species. That has an impact in two main factors required for quantification: the detection of the element of interest and the matrix correction. For regions with single NPs and ionic species, the matrix would likely correspond with that of the plant tissue but detection would be an issue, whereas for bigger aggregates, the matrix would be the element of the particle itself and the signal prone to self-absorption and detector saturation effects. In this sense, there is a need to develop reliable standardized protocols for quantification of NPs in plant tissues by SR-XFM and to determine the specific uncertainty of the method for this particular application.

\subsection{XAS data analysis}

XAS is divided in two regions according to the information that can be obtained, XANES and EXAFS. Since most applications of spatially resolved XAS involving the investigation of chemical modifications of NPs in plants have relied only on XANES, this section will focus on data analysis of this spectral region. XANES spectroscopy provides information about the valence state and coordination environment of the element of interest and about the molecular species present in the samples. Binding energies of the valence or- 

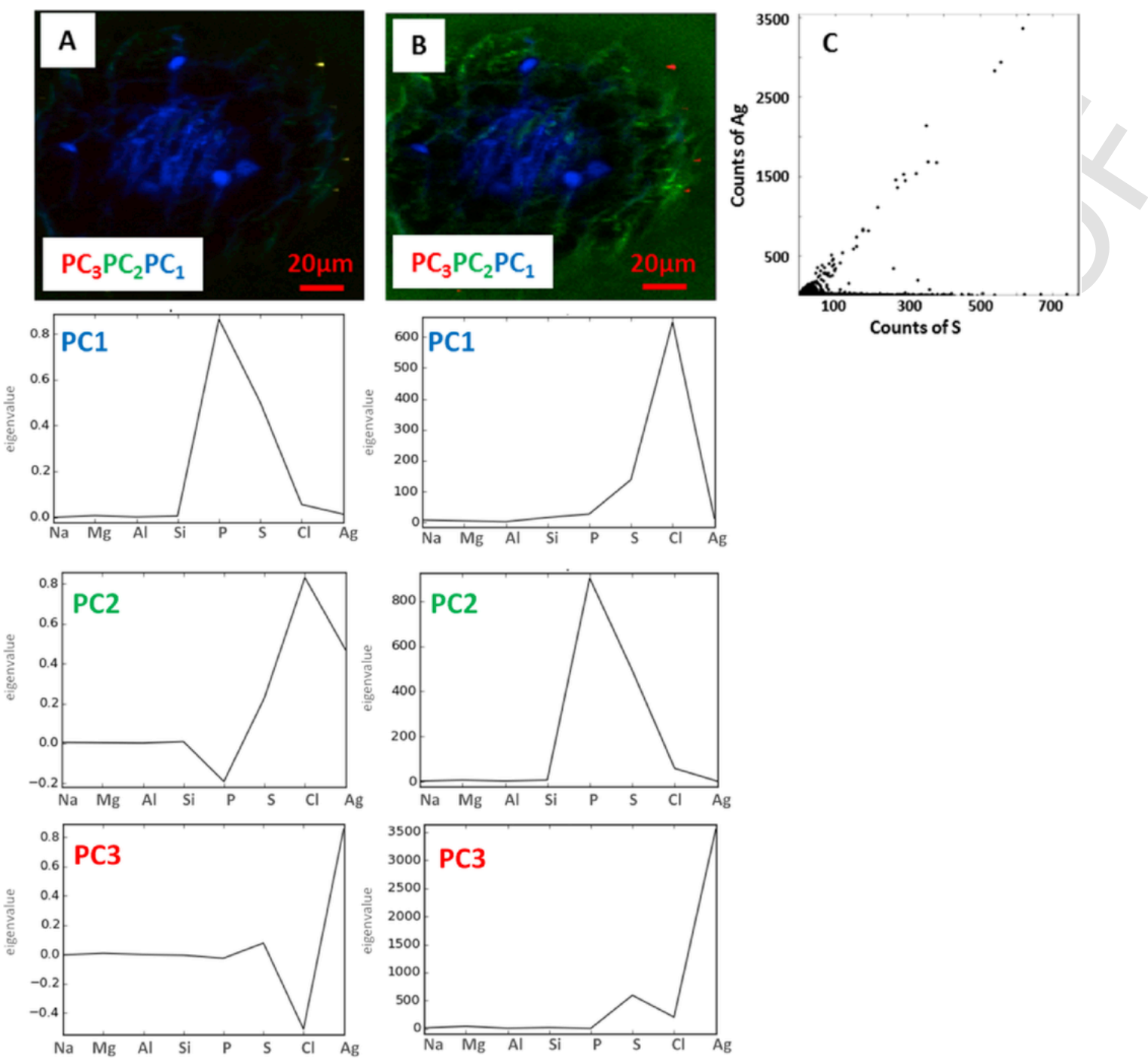

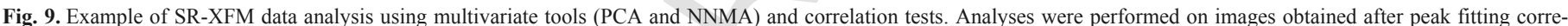

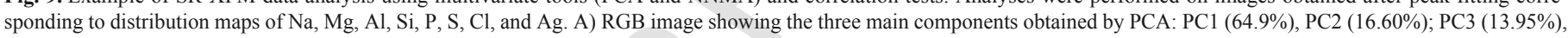

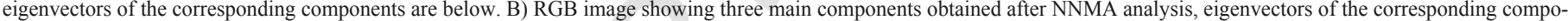
nents are below. C) Scatter plot of Ag vs S counts per pixel used to derive spatial correlation of these elements.

bitals are higher for more oxidized atoms. For a given element, the position of the absorption edge maximum or so-called white line increases by $1-3 \mathrm{eV}$ for each electron removed from the valence shell. For example, $\mathrm{CeL}_{\mathrm{III}}$-edge XANES spectra of $\mathrm{Ce}(\mathrm{III})$ compounds show a white line position at lower energy than Ce(IV) compounds (Fig. 11a). $\mathrm{CeO}_{2}$ NPs are considered very stable so Ce uptake and translocation by plants exposed to them are influenced by their possible chemical transformations. In a recent study, using Ce $\mathrm{L}_{\text {III }}$ XANES, Rui et al. (2015) showed the reduction of $\mathrm{CeO}_{2}$ NPs in the roots of Cucumis sativus and the translocation of $\mathrm{Ce}(\mathrm{III})$ compounds to the shoots of plants grown in a $\mathrm{P}$ deficient media.

The position of the white line is also influenced by the bonding environment of the absorbing atom allowing the identification of the first neighboring atoms around an element of interest. For a given valence state, the coordination to different ligands could lead to a difference in the position of the edge of about $1 \mathrm{eV}$. For example, for divalent $\mathrm{Zn}, \mathrm{Zn}-\mathrm{S}$ compounds show edges at lower energies than compounds containing $\mathrm{Zn}-(\mathrm{O} / \mathrm{N})$ bonds because $\mathrm{Zn}-\mathrm{S}$ bonds are longer. Taking the derivative of the XANES region helps in the inter- pretation of the absorption edge energy position especially when differences are small, as it is for $\mathrm{Zn}-(\mathrm{O} / \mathrm{N})$ and $\mathrm{Zn}-\mathrm{S}$ (Fig. 11c). Theoretically, the edge energy is expected to vary inversely with the square of the metal-ligand distance (Penner-Hahn, 2005). This could be useful, for example, in the study of the detoxification mechanisms of ZnO NPs in plants. XANES spectra collected in plant samples will show different features if $\mathrm{Zn}$ is mainly chelated by phytochelatins ( $\mathrm{Zn}$ $-\mathrm{S}$ bonds) or by organics acids ( $\mathrm{Zn}-\mathrm{O}$ bonds). XANES spectroscopy is also sensitive to crystalline arrangement for a same chemical composition. For example, the two polymorphs of $\mathrm{TiO}_{2}$, anatase and rutile, could be distinguished by the presence of characteristic features in the white line and in the second oscillation for Ti K-edge XANES (Fig. 11d). Using Ti K edge $\mu$ XANES, Servin et al. (2012) reported a preferential localization of rutile in leaves and trichomes of Cucumis sativus L. exposed to $\mathrm{TiO}_{2}$ NPs $(80 \%$ anatase $20 \%$ rutile). Another informative feature of the XANES region is the pre-edge. For the case of $\mathrm{TiO}_{2}$, anatase and rutile, could also be distinguished by the presence of 4 and 3 peaks in the pre-edge, respectively (Fig. 11e). Both anatase and rutile are formed by $\mathrm{Ti}-\mathrm{O}$ octahedra, but 

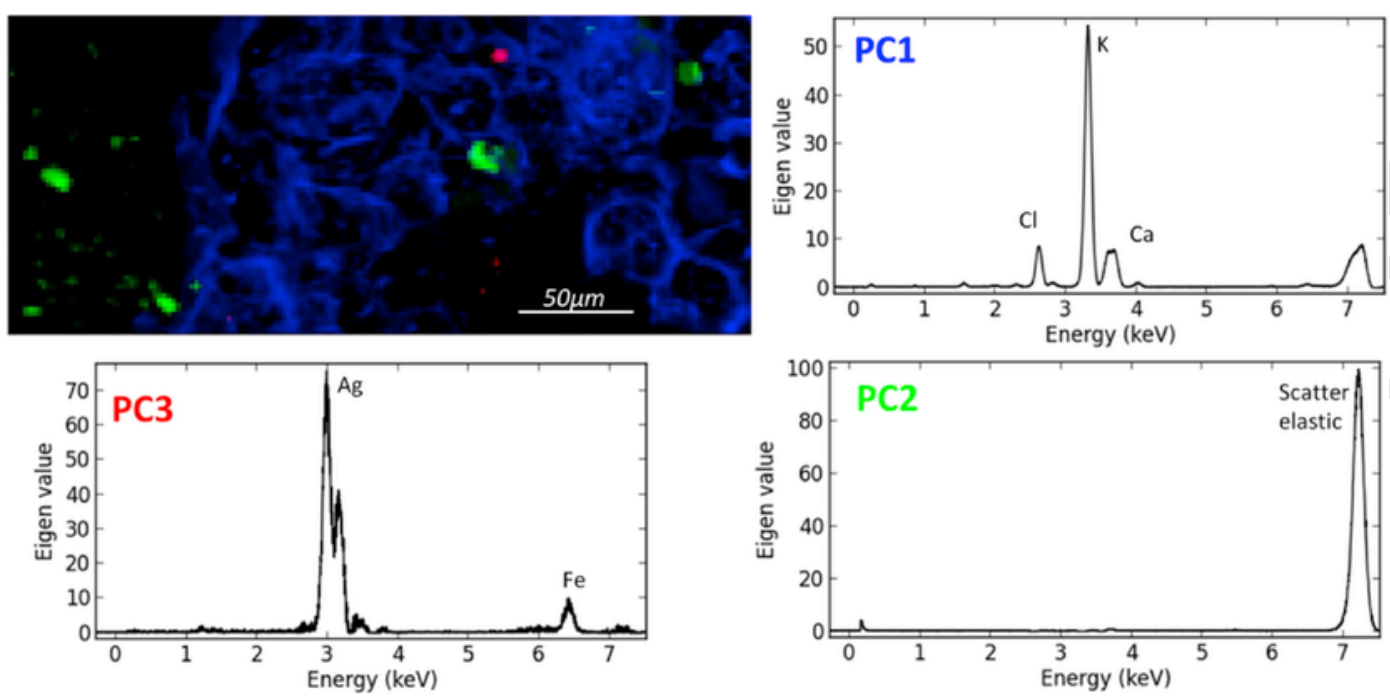

Fig. 10. NNMA analysis performed in full XRF spectra. SR- $\mu$ XFM map acquired at $7.2 \mathrm{keV}$ on Lactuca sativa L. leaf cross section exposed to Ag NPs by foliar deposition.
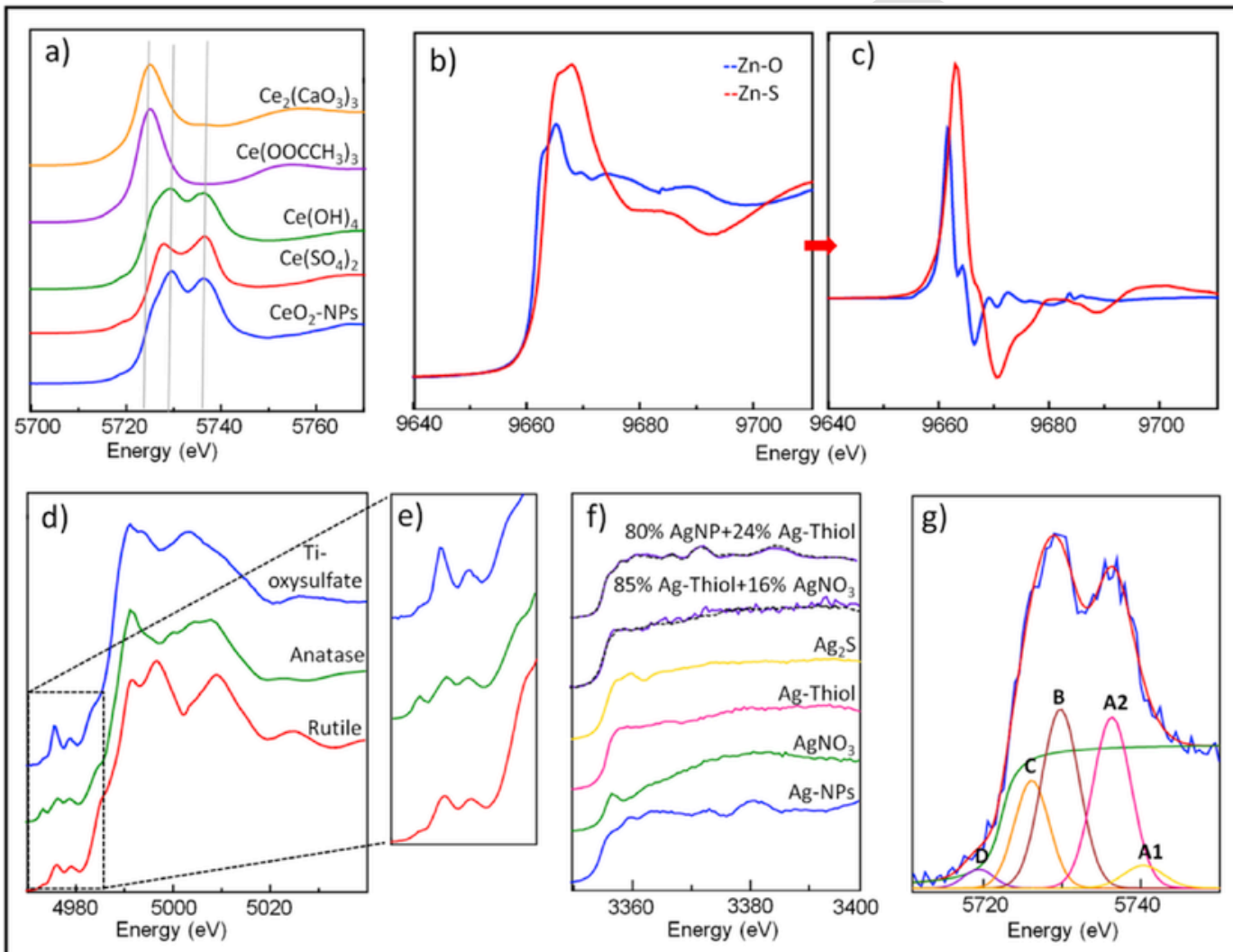

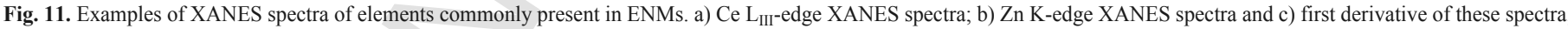

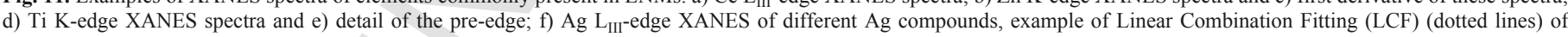

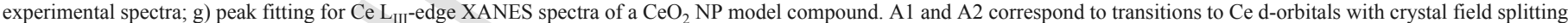

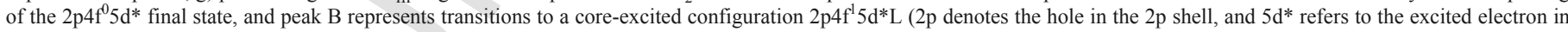

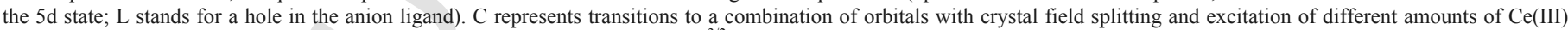
present in the samples. The pre-edge peak D is the result of a dipole-forbidden $2 \mathrm{p}^{3 / 2} 4 \mathrm{f}$ transition. All data analysis performed in Athena (Ravel and Newville, 2005).

also Ti compounds with tetrahedral symmetry can be identified by their characteristic pre-edge features as seen for Ti oxysulfate (Fig. 11e).

XANES data analysis requires the following steps: energy calibration, background subtraction, normalization, and data interpretation by comparison to reference spectra or by linear combination fitting (LCF). LCF is the most frequently used approach to identify and quantify specific molecular species present in unknown samples. This approach provides the distribution of chemical species in molar percentage of the target element in a sample. For example, Fig. $11 \mathrm{f}$ 
shows the Ag species found by LCF in Lactuca sativa L. after foliar exposure to Ag NPs, and evidences some chemical transformations (Larue et al., 2014a,b). This method uses a least-square algorithm to refine the sum of a given number of reference spectra to an experimental spectrum. The quality of the fits is estimated using the normalized sum of squares (NSS $=\Sigma(\mu \text { experimental }-\mu \text { fit })^{2} / \Sigma(\mu$ experimental $\left.)^{2} \times 100\right)$. In general, it is considered that the accuracy of the LCF method is $5-10 \%$ so a species is not included in the LCF unless it accounts for more than $5-10 \%$ of the total (Manceau et al., 2000; Kirpichtchikova et al., 2006). One of the most important points in this process is the library of reference spectra, which should be as exhaustive as possible.

An important assumption when using the LCF approach is the number of components used for the combinations, which corresponds to the number of chemical species present in the samples. Three to four components are generally used. Adding more components may improve the fit but may be meaningless if their contribution is close to the uncertainty of the method $(5-10 \%)$. A possible way to determine the number of components is the use of PCA on a set of unknown XANES spectra. PCA is a multivariate data analysis approach that finds the number of independent orthogonal eigenvectors (Principal Components) that better describe the variability of a data set by an approximation of weighted sums (Ressler et al., 2000; Manceau et al., 2014). The indicator value of Malinowski (IND) is often used to determine the number of statistically significant PCs needed to explain the variance of the dataset. Once the number of relevant PCs is determined, the XANES from known reference compound spectra are tested by Target Transformation (TT). This procedure fits (linear least squares fitting) each reference XANES spectrum using the components selected through the PCA procedure. A good fit validates the use of this reference for LCF of the unknown XANES data set. PCA together with TT allow the reduction of the dimensionality of the data set and the identification of the most probable components in the unknown XANES spectra.

Another approach is to treat Individual XANES spectra by peak fitting. In this procedure, the main features present in the XANES spectra are fitted by a set of Gaussian and arctangent functions. This methodology has been applied for the deconvolution of Ti pre-edge peaks by Larue et al. $(2012 \mathrm{a}, \mathrm{b})$ to study the speciation of $\mathrm{TiO}_{2} \mathrm{NPs}$ in wheat. Another example is the use of the double white line features at 5729 and $5737 \mathrm{eV}$ characteristic of $\mathrm{Ce}(\mathrm{IV}) \mathrm{L}_{\mathrm{III}}$-edge XANES spectra to further study small changes in the covalence between cerium and oxygen ligands (Fig. 8D) as used by Zhao et al. (2012) and Hernandez-Viezcas et al. (2013) to study the speciation of $\mathrm{CeO}_{2} \mathrm{NPs}$ in corn and soybean, respectively.

The various functions presented in this section are implemented in XAS data analysis packages such as Athena (Ravel and Newville, 2005), Sixpack (Webb, 2005) or the software developed in beamline 10.3.2 at the Advanced Light Source, Lawrence Berkeley National Lab (ALS-LBNL, Berkeley, CA) (https://sites.google.com/a/lbl.gov/ microxas-lbl-gov/software) and recently implemented in PyMca.

\section{Concluding remarks}

SR techniques have clearly provided significant insights for a better understanding of ENMs fate in plants, and conversely, this active research topic has surely stimulated the instrumental and technical developments. Multi-scale analyses are of high relevance and for this reason developments at present and future beamlines offering the possibility to perform micro and nano analysis at the same analytical platform on the same sample are envisioned. SR sources providing extremely brilliant beams will make possible to analyze higher num- ber of samples in less time with enhanced detection limits and improved lateral resolution. However, it will require a continuous development of highly sensitive and fast detectors. In the near future, 2D speciation in XRF mode (XANES mapping) will likely become a standard acquisition strategy, providing more statistically meaningful data sets to entangle the delicate aspects of plant/ENMs interactions. As presented in this review, sample preparation will continue to be critical for SR-XFM/XAS. Appropriate sample preparation protocols need to be further developed especially for nanoXFM in order to preserve ultrastructure and chemical speciation. Presently, the chosen sample preparation strategy depends on available sample environment at the beamline and auxiliary equipment available on site. Cryogenic set-ups are now available at several SR-XFM beamlines, this is highly beneficial particularly for speciation studies, and hence the number of studies in frozen-hydrated samples should increase in the near future. Investigations on fresh hydrated plant samples at room temperature is also expected to be increasingly used but its application for detailed localization at the nanoscale requires a tomographic approach which will likely present radiation damage issues. As already mentioned, the number of samples, spatial (more nano) and spectral resolution, and dimensionality of the data (e.g. hyperspectral XRF, or XANES mapping) are expected to keep increasing. Moreover, many existing beamlines offer complimentary techniques (XFM, XAS, XRD, FTIR, etc) at the same platform, and even simultaneous acquisition, which allow more complete understanding of the impacts of ENMs in plants. Hence, data analysis software that offers the adequate statistical tools for data mining and fast processing, with user friendly interfaces will be essential to assure successful data analysis and interpretation.

\section{Uncited references}

Donner et al., 2012; Martínez-Fernández et al., .

\section{Acknowledgements}

We thank the French program LabEx Serenade (11-LABX-0064) and the ESRF for providing a post doc fellowship for A.E.P. ISTerre is also part of Labex OSUG@2020 (ANR10 LABX56). Christian Olendrowitz is thanked for providing the sketch of the ID21 beamline.

\section{References}

Bandmann, V., Mueller, J.D., Koehler, T., Homann, U., 2012. Uptake of fluorescent nano beads into BY2-cells involves clathrin-dependent and clathrin-independent endocytosis. FEBS Lett. 586, 3626-3632.

Benn, T.M., Westerhoff, P., 2008. Nanoparticle silver released into water from commercially available sock fabrics. Environ. Sci. Technol. 42, 7025-7026.

Bittencourt, C., Ke, X., Van Tendeloo, G., Tagmatarchis, N., Guttmann, P., 2013. NEXAFS spectromicropscopy of suspended carbon nanohorns. Chem. Phys. Lett. 587, 85-87.

Biosolids, N.E., Association, R, 2007. A National Biosolids Regulation, Quality, End Use \& Disposal Survey. NEBRA, Tamworth, NH.

Castillo-Michel, H., Diaz-Sanchez, A., Martinez-Martinez, A., Hesse, B., 2016. Investigations of sulfur chemical status with synchrotron micro-focused X-ray fluorescence and X-ray absorption spectroscopy. Protein Pept. Lett. 23, 291-299.

Cañas, J.E., Long, M., Nations, S., Vadan, R., Dai, L., Luo, M., Ambikapathi, R., Lee, E.H., Olszyk, D., 2008. Effects of functionalized and nonfunctionalized single-walled carbon nanotubes on root elongation of select crop species. Environ. Toxicol. Chem. 27, 1922-1931.

Chwiej, J., Fik-Mazgaj, K., Szczerbowska-Boruchowska, M., Lankosz, M., Ostachowicz, J., Adamek, D., Simionovici, A., Bohic, S., 2005. Classification of nerve cells from substantia nigra of patients with Parkinson's disease and amyotrophic lateral sclerosis with the use of X-ray fluorescence microscopy and multivariate methods. Anal. Chem. 77, 2895-2900. 
Chwiej, J., 2010. The use of cluster and discriminant analysis in the investigations of the role of trace metals in the pathogenesis of Parkinson's disease. J. Trace Elem. Med. Biol. 24 (2), 78-88.

De Jonge, M.D., Ryan, C.G., Jacobsen, C.J., 2014. X-ray nanoprobes and diffraction-limited storage rings: opportunities and challenges of fluorescence tomography of biological specimens. J. Synchrotron Radiat. 21, 1031-1047.

Donner, E., Ryan, C., Howard, D., Zarcinas, B., Scheckel, K., McGrath, S., De Jonge, M., Paterson, D., Naidu, R., Lombi, E., 2012. A multi-technique investigation of copper and zinc distribution, speciation and potential bioavailability in biosolids. Environ. Pollut. 166, 57-64.

Donner, E., de Jonge, M.D., Kopittke, P.M., Lombi, E., 2013. Mapping element distributions in plant tissues using synchrotron X-ray fluorescence techniques. In: Maathuis, F.J.M. (Ed.), Plant Mineral Nutrients: Methods and Protocols. Springer science.

Fakra, S.C., Luef, B., Castelle, C.J., Mullin, S.W., Williams, K.H., Marcus, M.A., Schichnes, D., Banfield, J.F., 2015. Correlative cryogenic spectro-microscopy to investigate Selenium bioreduction products. Environ. Sci. Technol. http://dx.doi. org/10.1021/acs.est.5b01409.

Fayard, B., Pouyet, E., Berruyer, G., Bugnazet, D., Cornu, C., Cotte, M., De Andrade V., DiChiaro, F., Hignette, O., Kieffer, J., Martin, T., Papillon, E., Salome, M., Sole, V.A., 2013. The new ID21 XANES full-field end-station at ESRF. J. Phys. Conf. Ser. 425, 192001

Geranio, L., Heuberger, M., Nowack, B., 2009. The behavior of silver nanotextiles during washing. Environ. Sci. Technol. 43, 8113-8118.

Gräfe, M., Donner, E., Collins, R.N., Lombi, E., 2014. Speciation of metal(loid)s in environmental samples by X-ray absorption spectroscopy: a critical review. Anal. Chim. Acta 822, 1-22.

Guttmann, P., Bittencourt, C., Rehbein, S., Umek, P., Ke, X., Van Tendeloo, G., Ewels, C.P., Schneider, G., 2012. Nanoscale spectroscopy with polarized X-rays by NEXAFS-TXM. Nat. Photonics 6, 25-29.

Hackett, M.J., McQuillan, J.A., El-Assaad, F., Aitken, J.B., Levina, A., Cohen, D.D., Siegele, R., Carter, E.A., Grau, G.E., Hunt, N.H., 2011. Chemical alterations to murine brain tissue induced by formalin fixation: implications for biospectroscopic imaging and mapping studies of disease pathogenesis. Analyst 136, 2941-2952.

Hansson, M., Isaksson, M., Berg, G., 2008. Sample preparation for in vitro analysis of iodine in thyroid tissue using X-ray fluorescence. Cancer Inf. 6, 51-57.

Hare, D.J., New, E.J., de Jonge, M.D., McColl, G., 2015. Imaging metals in biology: balancing sensitivity, selectivity and spatial resolution. Chem. Soc. Rev. 44, 5941-5958.

Hartland, A., Lead, J.R., Slaveykova, V., O'Carroll, D., Valsami-Jones, E., 2013. The environmental significance of natural nanoparticles. Nat. Educ. 4, 7

Hernandez-Viezcas, J.A., Castillo-Michel, H., Servin, A., Peralta-Videa, J., Gardea-Torresdey, J., 2011. Spectroscopic verification of zinc absorption and distribution in the desert plant Prosopis juliflora-velutina (Velvet mesquite) treated with $\mathrm{ZnO}$ nanoparticles. Chem. Eng. J. 170, 346-352.

Hernandez-Viezcas, J.A., Castillo-Michel, H., Peralta-Videa, J., Andrews, J.C., Cotte, M., Rico, C., Peralta-Videa, J.R., Ge, Y., Priester, J.H., Holden, P., Gardea-Torresdey, J.L., 2013. In situ synchrotron X-ray fluorescence mapping and speciation of $\mathrm{CeO}_{2}$ and $\mathrm{ZnO}$ nanoparticles in soil cultivated soybean (Glycine max). ACS Nano 7 (2), 1415-1423

Hernandez-Viezcas, J.A., Castillo-Michel, H., Lopez-Moreno, M., Peralta-Videa, J., Gardea-Torresdey, J.L., 2016. Interactions between $\mathrm{CeO}_{2}$ nanoparticles and the desert plant mesquite: a Spectroscopy Approach. ACS Sust. Chem. Eng. 4 (3), $1187-1192$

Hinsinger, P., 1998. How do plant roots acquire mineral nutrients? chemical processes involved in the rhizosphere. Adv. Agron. 225-265.

Hong, J., Peralta-Videa, J.R., Gardea-Torresdey, J., 2013. Nanomaterials in agricultural production: benefits and possible threats? In: Shamim, N. (Ed.), Sustainable Nanotechnology and the Environment: Advances and Achievements. ACS, Whashington. ACS symposium series.

Hossain, Z., Mustafa, G., Komatsu, S., 2015. Plant responses to nanoparticle stress. Int J. Mol. Sci. 16, 26644-26653.

Isaure, M.P., Fayard, B., Sarret, G., Pairis, S., Bourguignon, J., 2006. Localization and chemical forms of cadmium in plant samples by combining analytical electron microscopy and X-ray spectromicroscopy. Spectrochim. Acta B 61, 1242-1252.

Isaure, M.-P., Huguet, S., Meyer, C.-L., Castillo-Michel, H., Testemale, D., Vantelon, D., Saumitou-Laprade, P., Verbruggen, N., Sarret, G., 2015. Evidence of various mechanisms of Cd sequestration in the hyperaccumulator Arabidopsis halleri, the non-accumulator Arabidopsis lyrata, and their progenies by combined synchrotron-based techniques. J. Exp. Bot. 66, 3201-3214.

Judy, J.D., Mcnear, D.H., Chen, C., Lewis, R.W., Tsyusko, O.V., Bertsch, P.M., Rao, W., Stegemeier, J.P., Lowry, G.V., Mcgrath, S.P., Durenkamp, M., Unrine, J. 2015. Nanomaterials in biosolids inhibit nodulation, shift microbial community composition, and result in increased metal uptake relative to bulk/dissolved metals. Environ. Sci. Technol. 49, 8751-8758.

Kaegi, R., Sinnet, B., Zuleeg, S., Hagendorfer, H., Mueller, E., Vonbank, R., Boller, M., Burkhardt, M., 2010. Release of silver nanoparticles from outdoor facades. Environ. Pollut. 158, 2900-2905.
Kirpichtchikova, T.A., Manceau, A., Spadini, L., Panfili, F., Marcus, M.A., Jacquet, T., 2006. Speciation and solubility of heavy metals in contaminated soil using $\mathrm{X}$-ray microfluorescence, EXAFS spectroscopy, chemical extraction, and thermodynamic modeling. Geochim. Cosmochim. Acta 70, 2163-2190.

Laborda, F., Bolea, E., Cepriá, G., Gómez, M.T., Jiménez, M.S., Pérez-Arantegui, J., Castillo, J.R., 2016. Detection, characterization and quantification of inorganic engineered nanomaterials: a review of techniques and methodological approaches for the analysis of complex samples. Anal. Chim. Acta 904, 10-32.

Lambrecht, E., Bare, J., Claeys, M., Chavatte, N., Bert, W., Sabbe, K., Houf, K., 2015. Transmission electron microscopy sample preparation protocols for the ultrastructural study of cysts of free-living protozoa. Biotechniques 58, 181-188.

Larue, C., Laurette, J., Herlin-Boime, N., Khodja, H., Fayard, B., Flank, A.-M., Brisset, F., Carriere, M., 2012b. Accumulation, translocation and impact of TiO2 nanoparticles in wheat (Triticum aestivum spp.): influence of diameter and crystal phase. Sci. Total Environ. 431, 197-208.

Larue, C., Veronesi, G., Flank, A.M., Surble, S., Herlin-Boime, Carriere, M., 2012a. Comparative uptake and impact of $\mathrm{TiO}_{2}$ nanoparticles in wheat and rapeseed. J. Toxicol. Env. Health. Part A 75, 722-734.

Larue, C., Castillo-Michel, H., Sobanska, S., Cecillon, L., Bureau, S., Barthes, V., Ouerdane, L., Carriere, M., Sarret, G., 2014a. Foliar exposure of the crop Lactuca sativa to silver nanoparticles: evidence for internalization and changes in Ag speciation. J. Hazard. Mat. 264, 98-106.

Larue, C., Castillo-Michel, H., Sobanska, S., Trcera, N., Sorieul, S., Cecillon, L., Ouerdane, L., Legros, S., Sarret, G., 2014b. Fate of pristine TiO2 nanoparticles and aged paint-containing $\mathrm{TiO}_{2}$ nanoparticles in lettuce crop after foliar exposure. J. Hazard. Mat. 273, 17-26.

Larue, C., Castillo-Michel, H., Stein, R.J., Fayard, B., Pouyet, E., Villanova, J., Magnin, V., Pradas del Real, A.E., Trcera, N., Legros, S., Sorieul, S., Sarret, G., 2016. Innovative combination of spectroscopic techniques to reveal nanoparticle fate in a crop plant. Spectrochim. Acta B 119, 17-24.

Lee, D.D., Seung, H.S., 1999. Learning the parts of objects by non-negative matrix factorization. Nature 401 (6755), 788-791.

Leroux, O., Leroux, F., Bellefroid, E., Claeys, M., Couvreur, M., Borgonie, G., Van Hoorebeke, L., Masschaele, B., Viane, R., 2009. A new preparation method to study fresh plant structures with X-ray computed tomography. J. Microsc. 233, 1-4.

Li, L., Sillanpää, M., Tuominen, M., Lounatmaa, K., Schultz, E., 2013. Behavior of titanium dioxide nanoparticles in Lemna minor growth test conditions. Ecotoxicol. Environ. Saf. 88, 89-94.

Lombi, E., Scheckel, K.G., Kempson, I.M., 2011. In situ analysis of metal(loid)s in plants: state of the art and artefacts. Environ. Exp. Bot. 72, 3-17.

Ma, C., White, J.C., Dhankher, O.P., Xing, B., 2015a. Metal-based nanotoxicity and detoxification pathways in higher plants. Environ. Sci. Technol. 49, 7109-7122.

Ma, Y., Zhang, P., Zhang, Z., He, X., Li, Y., Zhang, J., Zheng, L., Chu, S., Yang, K., Zhao, Y., Chai, Z., 2015b. Origin of the different phytotoxicity and biotransformation of cerium and lanthanum oxide nanoparticles in cucumber. Nanotoxicol 9, 262-270.

Majumdar, S., Peralta-Videa, J.R., Bandyopadhyay, S., Castillo-Michel, H., Hernandez-Viezcas, J.-A., Sahi, S., Gardea-Torresdey, J.L., 2014. Exposure of cerium oxide nanoparticles to kidney bean shows disturbance in the plant defense mechanisms. J. Hazard. Mat. 278, 279-287.

Majumdar, S., Peralta-Videa, J.R., Castillo-Michel, H., Hong, J., Rico, C.M., Gardea-Torresdey, J.L., 2012. Applications of synchrotron micro-XRF to study the distribution of biologically important elements in different environmental matrices: a review. Anal. Chim. Acta 28, 1-16.

Manceau, A., Lanson, B., Schlegel, M.L., Harge, J.C., Musso, M., Eybert-Berard, L., Hazemann, J.-L., Chateigner, D., Lamble, G.M., 2000. Quantitative Zn speciation in smelter-contaminated soils by EXAFS spectroscopy. Am. J. Sci. 300, 289-343.

Manceau, A., Marcus, M., Lenoir, T., 2014. Estimating the number of pure chemical components in a mixture by X-ray absorption spectroscopy. J. Synchrotron Rad. 21, 1140-1147.

Martinez-Criado, G., Villanova, J., Tucoulou, R., Salomon, D., Suuronen, J.-P., Laboure, S., Guilloud, C., Valls, V., Barrett, R., Gagliardini, E., Dabin, Y., Baker, R., Bohic, S., Cohen, C., Morse, J., 2015. ID16B: a hard X-ray nanoprobe beamline at the ESRF for nano-analysis. J. Synchrotron Radiat. 23, 344-352.

Martínez-Fernández, D., Vítková, M., Michálková, Z., Komárek, M., Engineered nanomaterials for phytoremediation of metal/metalloids contaminated soils: implications for plant physiology. In Anasari, A.A., Gill, S.S., Gill, R., Lanza, G.R., Newman, L. (Eds.) Phytoremediation: Management of Environmental Contaminants. Springer Intl, Switzerland.

McDonald, K.L., 2009. A review of high-pressure freezing preparation techniques for correlative light and electron microscopy of the same cells and tissues. J. Microsc. 235, 273-281

Moscatelli, A., Ciampolini, F., Rodighiero, S., Onelli, E., Cresti, M., Santo, N., Idilli, A., 2007. Distinct endocytic pathways identified in tobacco pollen tubes using charged nanogold. J. Cell Sci. 120, 3804-3819.

Muñoz, M., De Andrade, V., Vidal, O., Lewin, E., Pascarelli, S., Susini, J., 2006. Redox and speciation micromapping using dispersive X-ray absorption spectroscopy: 
application to iron chlorite mineral of a metamorphic rock thin section. Geochem. Geophys. 7 (11), 1-10.

Osán, J., Kéri, A., Breitner, D., Fábián, M., Dähn, R., Simon, R., Török, S., 2014. Microscale analysis of metal uptake by argillaceous rocks using positive matrix factorization of microscopic X-ray fluorescence elemental maps. Spectrochim. Acta B 91, 12-23.

Peng, C., Duan, D., Xu, C., Chen, Y., Sun, L., Zhang, H., Yuan, X., Zheng, L., Yang, Y., Yang, J., 2015. Translocation and biotransformation of $\mathrm{CuO}$ nanoparticles in rice (Oryza sativa L.) plants. Environ. Pollut. 197, 99-107.

Penner-Hahn, J.E., 2005. Characterization of "spectroscopically quiet" metals in biology. Coord. Chem. Rev. 249, 161-177.

Perrin, L., Carmona, A., Roudeau, S., Ortega, R., Evaluation of sample preparation methods for single cell quantitative elemental imaging using proton or synchrotron radiation focused beams, J. Anal. At. Spectrom., 2015, 30, 2525-2532.Pickering, I., Gumaelius, L., Harris, H.H., Prince, R.C., Hirsch, G., Banks, J.A., Salt, D.E., George, G.N., 2006. Localizing the biochemical transformations of arsenate in hyperaccumulating fern. Environ. Sci. Technol. 40 (16), 5010-5014.

Porta, D., Lopez-Iglesias, C., 1998. A comparison of cryo- versus chemical fixation in the soil green algae Jaagiella. Tissue cell 30 (3), 368-376.

Pradas del Real, A.E., Castillo-Michel, H., Kaegi, R., Sinnet, B., Magnin, V., Findling, N., Villanova, J., Carrière, M., Santaella, C., Fernández-Martinez, A., Levard, C., Sarret, G., Fate of Ag-NPs in Sewage Sludge after Application on Agricultural Soils, Env. Sci Technol., 50 (4), 2016, 1759-1768. Punshon, T., Chen, S., Finney, L., Howard, L., Jackson, B.P., Karagas, M.R., Ornvold, K., 2015. High-resolution elemental mapping of human placental chorionic villi using synchrotron X-ray fluorescence spectroscopy. Anal. Bioanal. Chem. 407, 6839-6850.

Punshon, T., Hirschi, K., Yang, J., Lanzirotti, A., Lai, B., Guerinot, M.L., 2012. The role of CAX1 and CAX3 in elemental distribution and abundance in Arabidopsis seed. Plant Physiol. 158, 352-362.

Pushie, M.J., Pickering, I.J., Korbas, M., Hackett, M.J., George, G.N., 2014. Elemental and chemically specific X-ray fluorescence imaging of biological systems. Chem. Rev. 114, 8499-8541.

Ravel, B., Newville, M., 2005. ATHENA, ARTEMIS, HEPHAESTUS: data analysis for X-ray absorption spectroscopy using IFEFFIT. J. Synchrotron Rad. 12, 537-541.

Reich, E.S., 2013. Ultimate upgrade for US synchrotron. Nature 501, 148-149.

Ressler, T., Wong, J., Roos, J., Smith, I.L., 2000. Quantitative speciation of Mn-bearing particulates emitted from autos burning (methylcyclopentadienyl)manganese tricarbonyl-added gasolines using XANES spectroscopy. Environ. Sci. Technol. 34, 950-958.

Richter, T., Biel, S., Sattler, M., Wenck, H., Wittern, K.P., Wiesendanger, R., Wepf, R., 2007. Pros and cons: cryo-electron microscopic evaluation of block faces versus cryo-sections from frozen-hydrated skin specimens prepared by different techniques. J. Microsc. 225, 201-207.

Rico, C.M., Majumdar, S., Duarte-Gardea, M., Peralta-Videa, J.R., Gardea-Torresdey, J.L., 2011. Interaction of Nanoparticles with edible plants and their possible implications in the food chain. J. Agric. Food Chem. 59, 3485-3498.

Rico, C.M., Morales, M.I., McCreary, R., Castillo-Michel, H., Barrios, A.C., Hong, J., Tafoya, A., Lee, W.-Y., Varela-Ramirez, A., Peralta-Videa, J.R., 2013. Cerium oxide nanoparticles modify the antioxidative stress enzyme activities and macromolecule composition in rice seedlings. Environ. Sci. Technol. 47, 14110-14118.

Rousseau, R.M., 2001. Detection limit and estimate of uncertainty of analytical XRF results. Rigaku J. 18 (2), 33-47.

Rui, Y., Zhang, P., Zhang, Y., Ma, Y., He, X., Gui, X., Li.,Y., Zhang, J., Zheng, L., Chu S., Guo Z., Chai, Z., Zhao Y., Zhang, Z., Transformation of ceria nanoparticles in cucumber plants isinfluenced by phosphate. Env Poll. 198, 2015, 8-14. Ryan, C.G., Etschmann, B.E., Vogt, S., Maser, J., Harland, C.L., Van Achterbergh, E., Legnini, D., 2005. Nuclear microprobe-synchrotron synergy: towards integrated quantitative real-time elemental imaging using PIXE and SXRF. Nuc. Inst. Meth. Phys. Res. B231, 183-188.

Sabo-Attwood, T., Unrine, J.M., Stone, J.W., Murphy, C.J., Ghoshroy, S., Blom, D. Bertsch, P.M., Newman, L.A., 2012. Uptake, distribution and toxicity of gold nanoparticles in tobacco (Nicotiana xanthi) seedlings. Nanotoxicol 6, 353-360.

Salomé, M., Cotte, M., Baker, R., Barrett, R., Benseny-Cases, N., Berruyer, G., Bugnazet, D., Castillo-Michel, H., Cornu, C., Fayard, B., 2013. The ID21 scanning X-ray microscope at ESRF. J. Phys. Conf. Ser. 182004. IOP Publishing.

Sarret, G., Pilon Smits, E., Castillo Michel, H., Isaure, M., Zhao, F., Tappero, R., 2013 Use of synchrotron-based techniques to elucidate metal uptake and metabolism in plants. Adv. Agron. 119, 1-82.

Sarret, G., Willems, G., Isaure, M.-P., Marcus, M.A., Fakra, S.C., Frerot, H., Pairis, S., Geoffroy, N., Manceau, A., Saumitou-Laprade, P., 2009. Zinc distribution and speciation in Arabidopsis halleri x Arabidopsis lyrata progenies presenting various zinc accumulation capacities. New Phytol. 184, 581-595.

Schlesiger, C., Anklamm, L., Stiel, H., Malzer, W., Kanngießer, B., 2015. XAFS spectroscopy by an X-ray tube based spectrometer using a novel type of HOPG mosaic crystal and optimized image processing. J. Anal. At. Spectrom. 30, 1080-1085.
Schwab, F., Zhai, G., Kem, M., Turner, A., Schnoor, J., Wiesner, M., 2016. Barriers, pathways and processes for uptake, translocation and accumulation of nanomaterials in plants - critical review. Nanotox 10 (3), 257-278.

Servin, A.D., Castillo-Michel, H., Hernandez-Viezcas, J.A., Diaz, B.C., Peralta-Videa, J.R., Gardea-Torresdey, J.L., 2012. Synchrotron micro-XRF and micro-XANES confirmation of the uptake and translocation of $\mathrm{TiO}_{2}$ nanoparticles in cucumber (Cucumis sativus) plants. Environ. Sci. Technol. 46, 7637-7643.

Servin, A.D., Morales, M.I., Castillo-Michel, H., Hernandez-Viezcas, J.A., Munoz, B., Zhao, L., Nunez, J.E., Peralta-Videa, J.R., Gardea-Torresdey, J.L., 2013. Synchrotron verification of $\mathrm{TiO} 2$ accumulation in cucumber fruit: a possible pathway of $\mathrm{TiO}_{2}$ nanoparticle transfer from soil into the food chain. Environ. Sci. Technol. 47, 11592-11598.

Singh, S.P., Vogel-Mikuš, K., Vavpetič, P., Jeromel, L., Pelicon, P., Kumar, J., Tuli, R., 2014. Spatial X-ray fluorescence micro-imaging of minerals in grain tissues of wheat and related genotypes. Planta 240 (2), 277-289.

Schaller, J., Brackhage, C., Paasch, S., Brunner, E., Bäucker, E., Dudel, E.G., 2013. Silica uptake from nanoparticles and silica condensation state in different tissues of Phragmites australis. Sci. Total Environ. 442, 6-9.

Sharma, V.K., Filip, J., Zboril, R., Varma, R.S., 2015. Natural inorganic nanoparticles-formation, fate, and toxicity in the environment. Chem. Soc. Rev. 44, 8410-8423.

Smart, K.E., Smith, J.A., Kilburn, M.R., Martin, B.G., Hawes, C., Grovenor, C.R., 2010. High-resolution elemental localization in vacuolate plant cells by nanoscale secondary ion mass spectrometry. Plant J. 63 (5), 870-879.

Solé, V., Papillon, E., Cotte, M., Walter, P., Susini, J., 2007. A multiplatform code for the analysis of energy-dispersive X-ray fluorescence spectra. Spectrochim. Acta B 62, 63-68.

Sombke, A., Lipke, E., Michalik, P., Uhl, G., Harzsch, S., 2015. Potential and limitations of X-Ray micro-computed tomography in arthropod neuroanatomy: a methodological and comparative survey. J. Comp. Neurol. 523 (8), 1281-1295.

Stegemeier, J.P., Schwab, F., Colman, B.P., Webb, S.M., Newville, M., Lanzirotti, A., Winkler, C., Wiesner, M.R., Lowry, G.V., 2015. Speciation matters: bioavailability of silver and silver sulfide nanoparticles to Alfalfa (Medicago sativa). Environ. Sci. Technol. 49, 8451-8460.

Sun, T.Y., Gottschalk, F., hungerbuhler, K., Nowack, B., 2014. Probabilistic modelling of engineered nanomaterial emissions to the environment: a spatio-temporal approach. Environ. Poll. 185, 69-76.

Tylko, G., Mesjasz-Przybyłowicz, J., Przybyłowicz, W., 2007. In-vacuum micro-PIXE analysis of biological specimens in frozen-hydrated state. Nucl. Instr. Meth. Phys. Res. 260, 141-148.

Vogel-Mikuš, K., Pongrac, P., Pelicon, P., 2014. Micro-PIXE elemental mapping for ionome studies of crop plants. Int. J. PIXE 24, 217-233.

Vogel-Mikuš, K., Pongrac, P., Pelicon, P., Vavpetič, P., Povh, B., Bothe, H., Regvar, M., 2009. Micro-PIXE analysis for localization and quantification of elements in roots of Mycorrhizal metal-Tolerant plants. In: Varma, A., Kharkwal, A.C. (Eds.), Symbiotic Fungi: Principles and Practice. Springer Berlin Heidelberg, Berlin, Heidelberg, pp. 227-242.

Vavpetič, P., Vogel-Mikuš, K., Jeromel, L., Potočnik, N.O., Pongrac, P., Drobne, D., Tkalec, Ž.P., Novak, S., Kos, M., Koren, , 2015. Elemental distribution and sample integrity comparison of freeze-dried and frozen-hydrated biological tissue samples with nuclear microprobe. Nucl. Instr. Meth. Phys. Res. 348, 147-151.

Wang, P., Menzies, N.W., Lombi, E., Sekine, R., Blamey, F.P.C., Hernandez-Soriano, M.C., Cheng, M., Kappen, P., Peijnenburg, W.J., Tang, C., 2015. Silver sulfide nanoparticles $\left(\mathrm{Ag}_{2} \mathrm{~S}-\mathrm{NPs}\right)$ are taken up by plants and are phytotoxic. Nanotoxicol 9 (8), 1041-1049.

Wang, Z., Xie, X., Zhao, J., Liu, X., Feng, W., White, J.C., Xing, B., 2012. Xylemand phloem-based transport of $\mathrm{CuO}$ nanoparticles in maize (Zea mays L.). Environ. Sci. Technol. 46, 4434-4441.

Wang, P., Menzies, N.W., Lombi, E., McKenna, B.A., Johannessen, B., Glover, C.J., Kappen, P., Kopittke, P.M., 2013. Fate of ZnO nanoparticles in soils and cowpea (Vigna unguiculata). Environ. Sci. Technol. 47, 13822-13830.

Webb. S., SIXpack: agraphical user interface for XAS analysis using IFEFFIT, Phys. Scripta T115, 2005, 1011-1014.West, M., Ellis, A.T., Potts, P.J., Streli, C., Vanhoof, C., Wobrauschek, P., 2015. 2015 Atomic Spectrometry Update - a review of advances in X-ray fluorescence spectrometry and their applications. J. Anal. At. Spectrom. 30 (9), 1839-1889.

Yin, L.C., Espinasse, B., Colman, B.P., Auffan, M., Wiesner, M., Rose, J., Liu, J., Bernhardt, E., 2011. More than ions: the effects of silver nanoparticles on Lolium multiflorum. Environ. Sci. Technol. 45, 2360-2367.

Zhao, L., Peralta-Videa, J.R., Varela-Ramirez, A., Castillo-Michel, H., Li, C., Zhang, J., Aguilera, R.J., Keller, A.A., Gardea-Torresdey, J.L., 2012. Effect of surface coating and organic matter on the uptake of $\mathrm{CeO}_{2} \mathrm{NPs}$ by corn plants grown in soil: insight into the uptake mechanism. J. Hazard. Mat. 225, 131-138.

Zhao, L., Sun, Y., Hernandez-Viezcas, J.A., Servin, A.D., Hong, J., Niu, G., Peralta-Videa, J.R., Duarte-Gardea, M., Gardea-Torresdey, J.L., 2013. Influence of $\mathrm{CeO}_{2}$ and $\mathrm{ZnO}$ nanoparticles on cucumber physiological markers and bioaccumulation of Ce and Zn: a life cycle study. J. Agric. Food Chem. 61, 11945-11951. 
Zhao, L.J., Peralta-Videa, J.R., Rico, C.M., Hernandez-Viezcas, J.A., Sun, Y.P., Niu, G.H., Servin, A., Nunez, J.E., Duarte-Gardea, M., Gardea-Torresdey, J.L., 2014 $\mathrm{CeO}_{2}$ and $\mathrm{ZnO}$ nanoparticles change the nutritional qualities of Cucumber $(\mathrm{Cu}-$ cumis sativus). J. Agric. Food Chem. 62, 2752-2759.

Zhao, L., Sun, Y., Hernandez-Viezcas, J.A., Hong, J., Majumdar, S., Niu, G., Duarte-Gardea, M., Peralta-Videa, J.R., Gardea-Torresdey, J.L., 2015. Monitoring the en- vironmental effects of $\mathrm{CeO}_{2}$ and $\mathrm{ZnO}$ nanoparticles through the life cycle of corn (Zea mays) plants and in situ $\mu$-XRF mapping of nutrients in kernels. Environ. Sci. Technol. 49, 2921-2928.

Zohdi, V., Whelan, D.R., Wood, B.R., Pearson, J.T., Bambery, K.R., Black, M.J.,

2015. Importance of tissue preparation methods in FTIR micro-spectroscopical analysis of biological tissues: 'Traps for new users'. Plos One 10, e0116491. 